The AstrophySiCAL JourNaL, 454:531-544, 1995 November 20 (C) 1995. The American Astronomical Society. All rights reserved. Printed in U.S.A

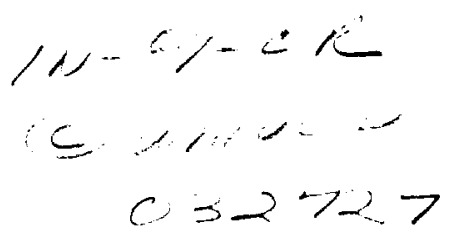

\title{
NEW OBSERVATIONS OF SUBARCSECOND PHOTOSPHERIC BRIGHT POINTS ${ }^{1}$
}

\author{
T. E. BERGER \\ Stanford University, ERL 328, Stanford, CA 94305-4055 \\ C. J. Schrijver, R. A. Shine, T. D. Tarbell, and A. M. Title \\ Lockheed Palo Alto Research Laboratory, Department 9130, B252, 3251 Hanover Street, Palo Alto, CA 94304 \\ AND \\ G. SCHARMER \\ Stockholm Observatory, Swedish Royal Academy of Sciences, S-13336 Saltsjöbaden, Sweden \\ Received 1995 March 16; accepted 1995 May 31
}

\begin{abstract}
We have used an interference filter centered at $4305 \AA$ within the bandhead of the $\mathrm{CH}$ radical (the " $\mathrm{G}$ band") and real-time image selection at the Swedish Vacuum Solar Telescope on La Palma to produce very high contrast images of subarcsecond photospheric bright points at all locations on the solar disk. During the 6 day period of 1993 September 15-20 we observed active region NOAA 7581 from its appearance on the East limb to a near-disk-center position on September 20. A total of 1804 bright points were selected for analysis from the disk center image using feature extraction image processing techniques. The measured FWHM distribution of the bright points in the image is lognormal with a modal value of $220 \mathrm{~km}(0.30)$ and an average value of $250 \mathrm{~km}\left(0^{\prime \prime} 35\right)$. The smallest measured bright point diameter is $120 \mathrm{~km}\left(0{ }^{\prime \prime} 17\right)$ and the largest is $600 \mathrm{~km}(0 \prime 69)$. Approximately $60 \%$ of the measured bright points are circular (eccentricity $\sim 1.0$ ), the average eccentricity is 1.5 , and the maximum eccentricity corresponding to filigree in the image is 6.5 . The peak contrast of the measured bright points is normally distributed. The contrast distribution variance is much greater than the measurement accuracy, indicating a large spread in intrinsic bright-point contrast. When referenced to an averaged "quiet-Sun" area in the image, the modal contrast is $29 \%$ and the maximum value is $75 \%$; when referenced to an average intergranular lane brightness in the image, the distribution has a modal value of $61 \%$ and a maximum of $119 \%$. The bin-averaged contrast of G-band bright points is constant across the entire measured size range. The measured area of the bright points, corrected for pixelation and selection effects, covers about $1.8 \%$ of the total image area. Large pores and micropores occupy an additional $2 \%$ of the image area, implying a total area fraction of magnetic proxy features in the image of $3.8 \%$. We discuss the implications of this area fraction measurement in the context of previously published measurements which show that typical active region plage has a magnetic filling factor on the order of $10 \%$ or greater. The results suggest that in the active region analyzed here, less than $50 \%$ of the small-scale magnetic flux tubes are demarcated by visible proxies such as bright points or pores.
\end{abstract}

Subject headings: Sun: activity - Sun: faculae, plages - Sun: magnetic fields

\section{INTRODUCTION}

This paper presents the first results of an ongoing study of small-scale solar magnetic structures using the Swedish Vacuum Solar Telescope (SVST) on the island of La Palma, Spain. The phrase "small-scale magnetic structures" refers in this case to the subarcsecond photospheric features found around sunspots or in the "quiet" supergranulation network boundaries. Previous studies (Ramsey, Schoolman, \& Title 1977; Title, Tarbell, \& Topka 1987; Keller 1992) have shown that there is discernible detail in small-scale magnetic field structures down to the operational resolution limit of all current ground-based telescopes (about 0.20 ), and it is virtually certain that additional detail remains hidden at still smaller scales. Study of these small-scale structures is important because they have been identified as crucial in the overall evolution of magnetic flux on the Sun, both in local active region emergence and decay (Muller \& Mena 1987; Strous 1994a), and in the global-scale diffusion of flux associated with

\footnotetext{
${ }^{1}$ Based on observations taken at the Swedish Vacuum Solar Telescope, which is operated on the island of La Palma by the Swedish Royal Academy of Sciences at the Spanish Observatorio del Roque de los Muchachos of the Instituto de Astrofisica de Canarias.
}

the solar magnetic cycle (Wang, Nash, \& Sheeley 1989; Sheeley 1992). Motion of small-scale magnetic structures in the photosphere may also contribute to coronal heating through topological rearrangement of flux tubes (Van Ballegooijen 1986; Parker 1990; Muller et al. 1994).

The direct method of imaging the solar magnetic field is through Zeeman effect magnetograms, which are typically produced either by slit-scanning spectroheliograms (Kitt Peak, ZIMPOL, Mount Wilson, Stanford) or multifiltergram averaging (LPARL, Big Bear). Both of these methods are limited by instrumental spatial resolution and/or by temporal seeing variations between images and therefore exhibit somewhat lower spatial resolution than single-image observations. To maximize spatial resolution in studies of magnetic structures, it is therefore desirable to observe one of the visible-light signatures, or "proxies," of the magnetic field in a fast-exposure single image. Traditionally these proxies have been referred to by their appearance: bright small-scale field patches at the limb are called "faculae"; small bright filamentary features in active regions are termed "filigree"; individual bright points in the filigree or faculae are referred to as "facular bright points"; small bright features in the quiet photospheric network are termed "network bright points"; and dark small-scale mag- 
netic structures are termed " micropores," "knots," or "holes." The smallest field proxies are the individual subarcsecond facular or network bright points (referred to hereafter simply as "bright points") which vary in observed angular size from 0"2 to 0"8 (Dunn \& Zirker 1973; Mehltretter 1974; Muller \& Keil 1983; Yi \& Engvold 1993). Although the physical mechanism behind the intrinsic contrast of bright points is uncertain (Solanki 1993), their first order association with magnetic fields is well established (Title et al. 1992; Keller 1992; Yi \& Engvold 1993), and they are accepted as reliable tracers of some fraction of the small-scale magnetic field elements or "flux tubes."

The problem with relying on bright points as small-scale field tracers is that in continuum wavelengths near disk center they exhibit low or even negative contrast values which depend critically on spatial resolution (Foukal, Duvall, \& Gillespie 1981 ; Foukal \& Fowler 1984; Hirayama, Hamana, \& Mizugaki 1985; Topka, Tarbell, \& Title 1992). They begin to have a small positive contrast in the continuum at a disk position of between $\mu=0.94$ (heliocentric angle $20^{\circ}$; Topka et al. 1992) and $\mu=0.76$ (heliocentric angle $40^{\circ}$; Foukal et al. 1981) but are not easily observable until near the limb. First attempts to image faculae at disk center were made by Sheeley (1969) using spectroheliograms in the center of temperature-sensitive absorption lines -effectively imaging in the highest levels of the photosphere. Subsequent experiments with spectroheliograms in the violet $3883 \AA$ bandhead of the $C N$ radical found that faculae were visible at disk center for many wavelengths within the bandhead. In a following study, Chapman (1970) substituted an interference filter centered in the $\mathrm{CN}$ bandhead for the spectroheliograph. The resulting short-exposure images of faculae showed an average contrast $(\Delta I / I)$ of $20 \%$ across the entire disk. Muller \& Roudier (1984) used a $10 \AA$ bandpass filter centered at $4308 \AA$ within the bandhead of the $\mathrm{CH}$ radical (known as the " $G$ band") in combination with high-speed photographic techniques to study bright points in the quiet photospheric network. Their results showed conclusively that broadband filters in molecular bandheads combined with very short exposure times give excellent bright point images for all disk locations.

\section{OBSERVATIONS}

The SVST is a $48 \mathrm{~cm} \mathrm{f} / 45$ doublet refractor telescope in a vacuum turret and tower configuration (Scharmer et al. 1985). The solar image formed by the doublet lens is relayed by three flat mirrors to a horizontal optical bench at the base of the tower. Active servo control of the third mirror is used for pointing and has recently been upgraded to incorporate correlation tracking of granulation. The prime-focus plate scale is $102 \mu \mathrm{m} \operatorname{arcsec}^{-1}$. The G-band filter (manufactured by Andover Corporation) is a dielectric interference filter centered at 4305 $\AA$ with a full width at half-maximum (FWHM) bandpass of 12 $\AA$. It is placed in the optical beam with a rotation about the vertical axis of less than $5^{\circ}$. The camera is a Kodak Megaplus 1.4 CCD camera run at room temperature. The Megaplus 1.4 has a $1360 \times 1036$ format with $6.4 \mu \mathrm{m}$ pixels and an 8 bit readout; the prime-focus image scale is 15.92 pixels arcsec ${ }^{-1}$ with a total field of view of approximately $65^{\prime \prime} \times 80^{\prime \prime}$. The CCD camera is controlled by a Digital Equipment Corporation (DEC) Alpha workstation; images are relayed to the Alpha via a custom interface board designed by DEC Research Laboratories, Paris. Minimum exposure time is $10 \mathrm{~ms}$. The frame readout time of the camera is $130 \mathrm{~ms}$, resulting in a maximum sustained frame rate of 7 frames $\mathrm{s}^{-1}$. A real-time contrast evaluation algorithm operates on a $256 \times 256$ pixel $\left(\sim 16^{\prime \prime} \times 16^{\prime \prime}\right)$ subframe of each image to quantify seeing blur (Scharmer 1989). The image with the highest subframe quality (i.e., the least seeing blur) in a $10 \mathrm{~s}$ sample of 70 frames is stored for later analysis. Time-series observations consisting of over 2000 frames and spanning $11 \mathrm{hr}$ have been achieved with this system (Simon et al. 1994).

Over the course of 6 days (1993 September 15-20), AR 7581 was imaged from its east limb appearance to a near-diskcenter position. Figure 1 (Plate 27) shows the best image obtained of AR 7581 on 1993 September 15 at 16:36 UT as it appeared near the east limb at a disk position of about $\mu=0.45$. The exposure time for the image was $15 \mathrm{~ms}$. The region is a two-spot complex, with the upper spot in the image beginning to fragment by a large light bridge through its center. Remnant penumbrae are seen on both spots. The appearance of the faculae in this image is typical of many previous observations of faculae in both continuum and other bandpasses, with the notable exceptions of very high spatial resolution (demonstrated by the resolution of some facular structures into 0.3 diameter bright points) and contrast. The larger faculae, as well as some of the bright points, are saturated in the 8 bit image. Figure 2 (Plate 28) shows the trailing part of the region as it appeared near disk center $(\mu=0.94)$ on 1993 September 20 at 14:38 UT. The exposure time for the image was $11 \mathrm{~ms}$. The fragmentation of the upper spot in Figure 1 has resulted in the line of pores across the top of the image, while the lower spot in Figure 1 has contracted uniformly to form the $5^{\prime \prime}$ diameter pore in the lower central region of Figure 2 (this evolutionary sequence is established by images from the intervening days, which are not shown here). It is interesting to note that while the upper spot decayed by fragmentation into a line of small pores, the lower spot simply decreased in size while remaining a coherent flux element.

The near-disk-center image of 1993 September 20 shown in Figure 2 exhibits the highest resolution of any of the images in the 6 day series. It is practically unaffected by seeing across the entire $65^{\prime \prime} \times 80^{\prime \prime}$ field of view and is one of the highest resolution images of photospheric bright points ever obtained. The characteristics of G-band images near disk center are well demonstrated by this image: the image rms contrast is $10.6 \%$, with a maximum bright-point contrast of $75 \%$ (relative to a quiet granulation average). Many isolated bright points whose observed FWHM diameters are $0.18(130 \mathrm{~km}$ on the Sun) are measured on the image. We note that this diameter is approximately equal to the FWHM diameter of the peak of the Airy function of the SVST at $4300 \AA$, which is significantly less than the Rayleigh limit diameter typically quoted as the "classical diffraction limit" $\left(00^{\prime \prime 23}\right.$ for the SVST at $4300 \AA$; Title et al. 1995). Several filigree structures are visible near the center of the image. All larger pores in the central area of the image are ringed by bright points, some of which are highly elongated in the radial direction. There is also an instance of a solitary micropore approximately $1^{\prime \prime} .5$ in length and 0.5 in width which is ringed entirely by bright points no larger than 0".2 FWHM. Where there are no pores but a high number density of bright points, such as in the upper central portion of Figure 2, the granulation texture is disturbed and contains many small irregularly shaped granules: an effect commonly referred to as "abnormal granulation" (Dunn \& Zirker 1973; Dunn, Mann, \& Simon 1973; Title et al. 1989; de Boer \& Kneer 1992). Figure 3 (Plate 29) is the central $200 \times 200$ pixel area of Figure 2 enlarged by a factor of 3 . In this image it can be seen that the 
filigree have fluted boundaries and are probably composed of strings of bright points in close proximity. The characteristic FWHM of the filigree is $0.2(\sim 150 \mathrm{~km})$, in general agreement with the filigree observations of Dunn \& Zirker (1973).

\section{ANALYSIS}

\subsection{Image Processing}

Analysis of the G-band data was performed on the September 20 image because it exhibits the highest resolution of the set and is nearest disk center. A bias-level correction and flatfielding process were first applied to the image. In order to enhance the bright points relative to the granulation, the calibrated image was deconvolved by a theoretical telescope modulation transfer function (MTF). Because this procedure introduces spurious high spatial frequency artifacts near sharp gradients in intensity (the well-known Gibbs phenomenon), the enhanced image was used only in segmenting the bright points from the granulation and was not used in subsequent measurements of bright-point contrast.

For separating the bright points from granulation a simple threshold operation on the raw image is unsuccessful; granulation contains local peaks of varying levels which are not distinguished from bright points by any single threshold value. Also, simple thresholding misses many bright points which are low contrast compared to the image mean intensity but locally high contrast (when compared with the surrounding intergranular intensities). A more sophisticated method was devised for this study based on a "blob finding" algorithm originally developed by A. Rosenfeld (Tomita 1990). The algorithm computes the following quantity at each pixel in the image:

$$
\begin{aligned}
B(x, y)= & \frac{1}{(2 M+1)^{2}} \sum_{u=x-M}^{x+M} \sum_{v=y-M}^{y+M} I(u, v) \\
& -\frac{1}{(2 N+1)^{2}} \sum_{u=x-N}^{x+N} \sum_{v=y-N}^{y+N} I(u, v),
\end{aligned}
$$

where $M$ and $N$ are integers, $M<N$, and $I(x, y)$ is the intensity of the image at the pixel location $(x, y)$. The operator can be used to detect either bright blobs ( $B$ positive) or dark blobs ( $B$ negative). We find that at the plate scale of the images shown here (15.92 pixel arcsec $^{-1}$ ), $M=1$ (corresponding to a $3 \times 3$ square neighborhood, 0.18 across) and $N=3$ (corresponding to a $7 \times 7$ square neighborhood, 0.43 across) give good initial enhancement of the bright points over the background granulation. The resulting "blob enhanced" image is further processed with an unsharp-mask algorithm to sharpen the boundaries of the bright structures. A threshold operation is then performed which results in a binary image which has the value unity at the locations of bright points (and some residual granulation peaks) and zero elsewhere. One copy of the binary image is "opened" (Haralick, Sternberg, \& Zhuang 1987) using dilation and erosion processing with a 5 pixel " +" kernel to eliminate most of the larger residual granulation noise and separate proximal bright points. A second copy of the binary image is median filtered in a $3 \times 3$ pixel neighborhood. The two processed images are then added using a Boolean "OR" operation to create the binary bright-point map. Finally, most of the remaining granulation peaks are subtracted manually from the binary map. In the case of the September 20 image, 252 granulation peaks, representing about $12 \%$ of the total objects in the binary map, were removed manually. Figure 4 (Plate 30 ) is an overlay image consisting of the $200 \times 200$ pixel area of Figure 3 with the bright-point binary map subtracted. The figure shows that the map successfully outlines the contour of the bright points with no noticeable artificial structure added. However, at the scale of Figure 4, pixelation of the structures is evident in all cases.

The pixelation evident in Figure 4 indicates that the binary map will not accurately represent the area or the total number of the smallest bright points in the image. The smallest bright points one can identify by eye in the original image are circular bright points with FWHM diameters of only 3-4 pixels. If they are among the lower contrast bright points, the thresholding operation will discriminate against these objects and they will not be included in the binary map. By a random survey of a dense bright-point area in Figure 2, it was determined that about $15 \%$ of the smallest bright points that are visually identifiable were not included in the binary map. The smallest bright points which are of high enough contrast to be included by the threshold are eroded by the opening process to the point that their pixel representation is equal to the kernel. The 5 pixel " + " kernal underestimates the area of a 3 pixel diameter circle by about $40 \%$. Therefore, while the final binary map is an accurate estimation of the position of most bright-points centroids, it posseses selection and pixelation errors which must be compensated for in the analysis.

Following the completion of the binary map, each object in the binary map is extracted for individual measurement. The extraction process used for this purpose is a nearest-neighbor algorithm which develops contiguous, 4-connected pixel sets. The routine takes as input the full binary map and outputs the coordinates of each set of 5 or more pixels which comprise a single object. The 5 pixel cutoff was chosen because most of the granulation noise objects were found to be represented in the binary map by only 3-4 pixels, and the manual removal of granulation noise cannot be assumed perfect. For each extracted object, the routine tabulates the total number of pixels (equivalent to area in units of square pixels), the location of the centroid, and a list of coordinates of the pixels comprising the object. By referencing the given pixel coordinates in the G-band image, the contrast of a single bright point can be measured. We determine both peak contrast and mean contrast for each object in the binary map. Peak contrast is defined as the following ratio:

$$
C(n)_{\text {peak }} \equiv \frac{I(n)_{\text {peak }}-I_{\text {ref }}}{I_{\text {ref }}},
$$

$I(n)_{\text {peak }}$ is the peak intensity in the G-band image of the $n$th object in the binary map and $I_{\text {ref }}$ is some reference intensity in the image. Similarly, the mean contrast of a given object, $C(n)_{\text {mean }}$, is defined by substituting the mean of all intensity values from the G-band image, $I(n)_{\text {mean }}$, for $I(n)_{\text {peak }}$ in equation (2).

\subsection{Image Statistics}

A total of 1804 objects composed of 5 or more pixels were extracted from the binary map. The primary measurement in the analysis sequence is the bright point size. We quantify bright point size primarily on the basis of the FWHM intensity diameter. The FWHM measurement is taken in the direction of the smallest dimension of a given bright structure so that the measurement is not biased by the shape of the bright point. For example, the long filigree are measured across their narrow dimension and not in their long direction. The measurements are performed by a semiautomated routine which 
takes as input the centroid location of each object in the binary map and returns as output a 32 pixel $\left(\sim 2^{\prime \prime}\right)$ long cut through the centroid in the direction which approximately minimizes the measured diameter. A nonlinear least-squares Gaussian fit which includes a second-order polynomial correction to the surroundings of the bright point is then applied to the extracted profile. The tabulated diameter is given by the bestfit Gaussian FWHM value. Figure 5 shows a typical example of a bright-point profile taken from Figure 2, and its Gaussian fit. The granulation and intergranular lane intensities are enhanced in the region surrounding this particular bright point, as is evidenced by the $0 \%$ contrast (with respect to an averaged quiet-Sun area) of the intergranular lane. In addition, some of the objects in this area were found to be too close to other bright points to allow a good profile cut to be made. Therefore, only 1234 bright structures out of the total 1804 in the binary map were measured for their FWHM values.

Figure 6 shows the histogram of the 1234 measured FWHM values of the bright points and filigree. The distribution is positively skewed and clearly non-Gaussian. It is well fitted by a second-order polynomial function on the $\log -\log$ equivalent of Figure 6 (shown as the solid curve in the figure), implying that the FWHM distribution is lognormal. The lognormal distribution has previously been used to describe the size distribution of sunspot umbrae (Bogdan et al. 1988) and can be heuristically derived from a fragmentation mechanism; it is therefore not surprising to find its application in small-scale magnetic structure as well. The lognormal distribution gives a modal FWHM value of $220 \mathrm{~km}\left(0^{\prime \prime} 3\right)$ and an average of $250 \mathrm{~km}$ $(0 " 35)$. The smallest FWHM value measured is $120 \mathrm{~km}\left(0^{\prime \prime} .17\right)$. The largest bright point measured has a FWHM of $600 \mathrm{~km}$ (0.69).

Under visual inspection, the shape of the majority of the bright points is found to be nearly circular. However, especially near pores, many bright points are significantly elongated. The filigree, which are evidently strings of circular bright points in close proximity, also appear elongated. It is therefore of interest to determine if a shape quantification can indicate whether the smaller elongated structures are consistent with a line of smaller circular points, as in the case of the filigree. We quantify shape by a principal-axis transformation of the covariance matrix associated with each individual structure in the binary map. This transformation gives the major and minor axes of the ellipse which best describes the structure. It is found that the eccentricity derived from the elliptical approximation to the bright points is a good measure of relative "elongation " of the bright points. Figure 7 shows the histogram of derived eccentricity for all structures in the binary map. Approximately $60 \%$ of the bright points measured are near-circular, with an eccentricity less than 1.2. The average eccentricity is 1.5 , and the maximum is 6.5 . The eccentricity shows a positive correlation with bright-point area (i.e., the largest eccentricities correspond to the filigree); however, this measure is not conclusive for determining whether the smaller eccentric bright points are consistent with the shape of filigree. Analysis in this area is ongoing.

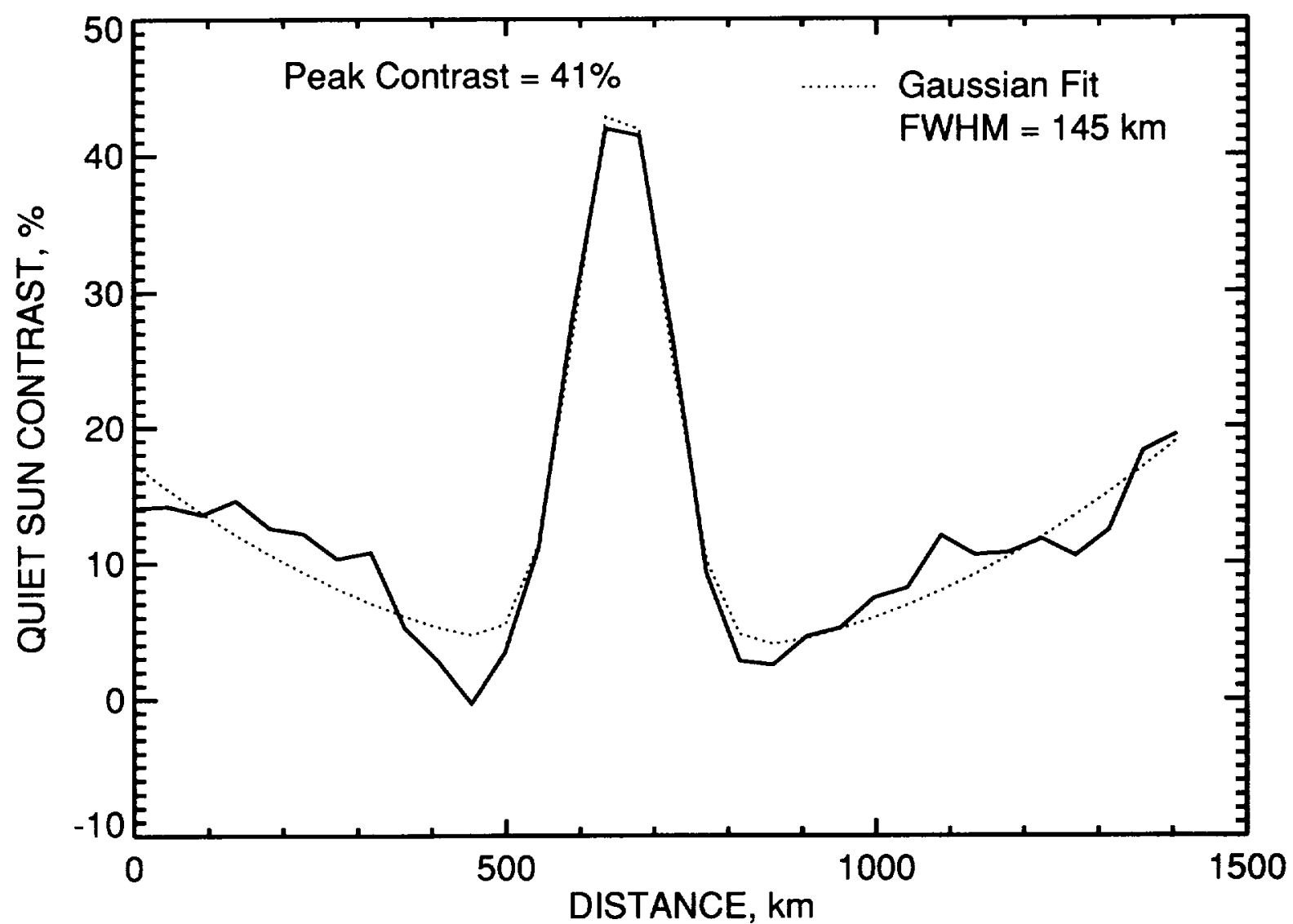

Fig. 5.-Profile cut through a typical bright point in the image of Fig. 2 . The cut is 32 pixels $\left(2^{\prime \prime}, \sim 1400 \mathrm{~km}\right)$ in length and has been de-trended using a second-order polynomial fit. The Gaussian fit includes a second-order fit to the surrounding profile. 


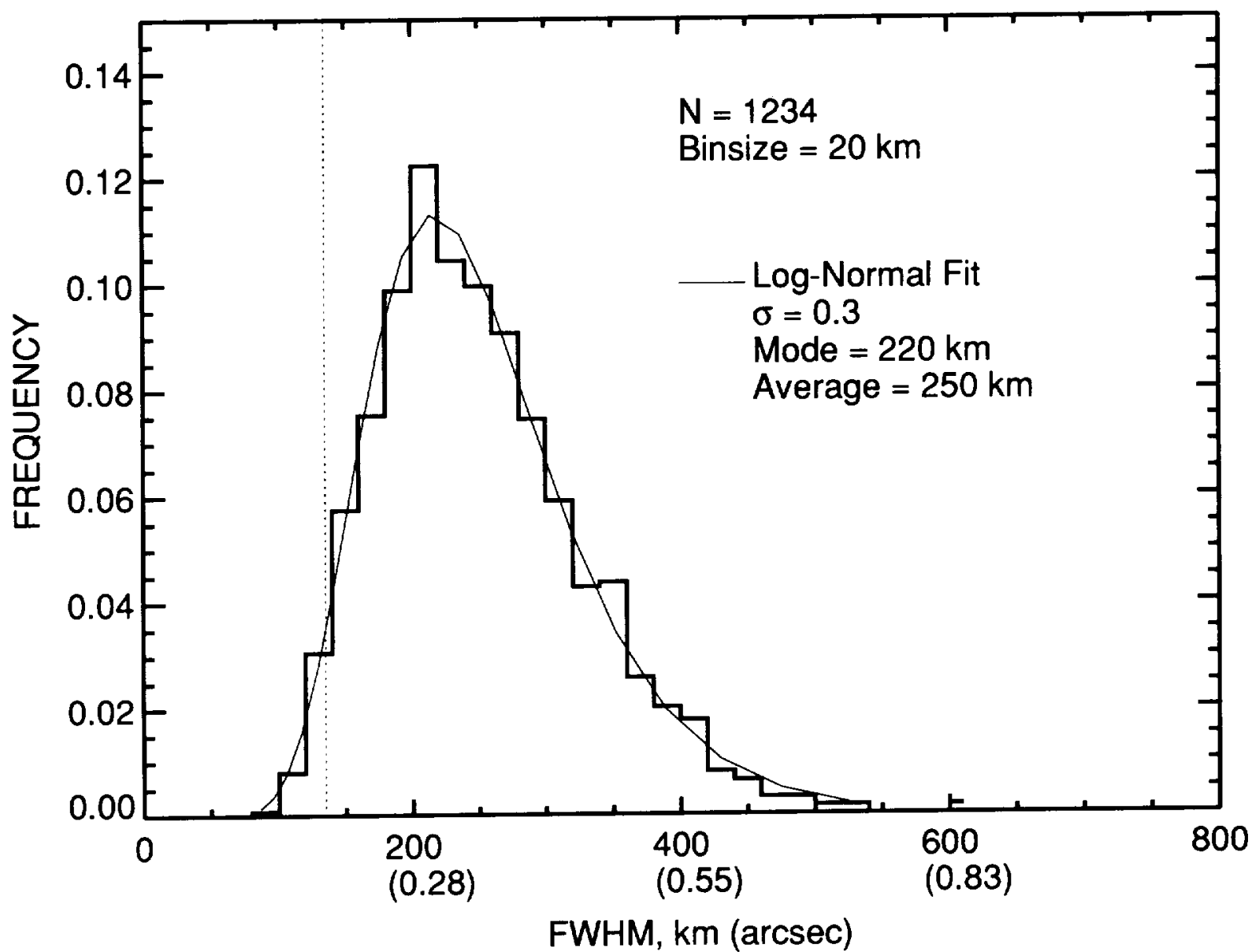

Fig. 6.-FWHM diameter histogram for 1234 bright points in Fig. 2. All measurements are performed to give the least FWHM for a given structure; the filigree are measured perpendicular to their long axis. The dashed vertical line is the theoretical Rayleigh resolution limit of the SVST.

The peak contrast histogram of the bright points is shown in Figure 8. Two reference intensities are used in the figure: a $100 \times 100$ pixel average of "quiet-Sun" granulation (granulation showing no evidence of bright points or pores) and an average intergranular lane intensity calculated by sampling along several intergranular lanes both near to and far from bright points. The peak contrast distribution is roughly Gaussian with an average value of $31 \%$ relative to quiet granulation and $64 \%$ relative to the intergranular lanes. The standard deviation of the peak contrast distribution is $11.2 \%$. The maximum measured quiet-Sun contrast is $75 \%(119 \%$ contrast relative to the lanes). We include the intergranular lane reference to emphasize that local surroundings of bright points can greatly influence their visibility. As an extreme example, the lowest intensity bright structure measured has a peak contrast of $-19 \%$ relative to the quiet granulation reference intensity; such a structure would certainly be called a dark point were it not for the fact that this particular bright point is located inside a pore: relative to the average intergranular lane it has a contrast of $1 \%$, and relative to its immediate local surroundings (the pore) the same structure has a contrast of $23 \%$, making it easily visible in the image.

The sensitivity of bright-point contrast to the immediate local surroundings points out the main problem intrinsic to low spatial resolution measurements of bright points: when averaged with their local surroundings on scales of 0.5 or more, the contrast of bright points is severely decreased and is often negative. This point is demonstrated by convolving the September 20 image with a 0 ". 5 Gaussian kernel, overlaying the original bright-point binary map, and then carrying out the same analysis that led to Figure 8 . The modal peak contrast of the bright points relative to the quiet granulation in the artificial image decreases to $15 \%$, and many of the bright points which are easily identified in the original image are smeared into very low contrast features. The primary cause of the contrast decrease is the local averaging of small bright points with the dark intergranular lanes (Title et al. 1995).

Figure 9 plots the peak contrast relative to quiet granulation of the bright points against their measured FWHM size. The solid line in the plot is the average contrast as a function of size computed by averaging the contrast in $50 \mathrm{~km}$ FWHM size bins. The bin-averaged contrast shows that bright-point contrast in the $G$ band is not dependent on size to any measurable degree. In addition, the data exhibit an extremely large scatter about the mean values for sizes up to $450 \mathrm{~km}$. The maximum quiet-Sun contrast of $75 \%$ occurs for an intermediate-sized bright point with a FWHM of about $170 \mathrm{~km}(0 " 23)$. The data in Figure 9 were used to construct a two-dimensional histogram of contrast versus FWHM size. Shown in Figure 10 are four vertical sections through the two-dimensional histogram, resulting in contrast histograms for FWHM bins of 125-175, $175-225,225-275$, and $275-325 \mathrm{~km}$, respectively. The central 


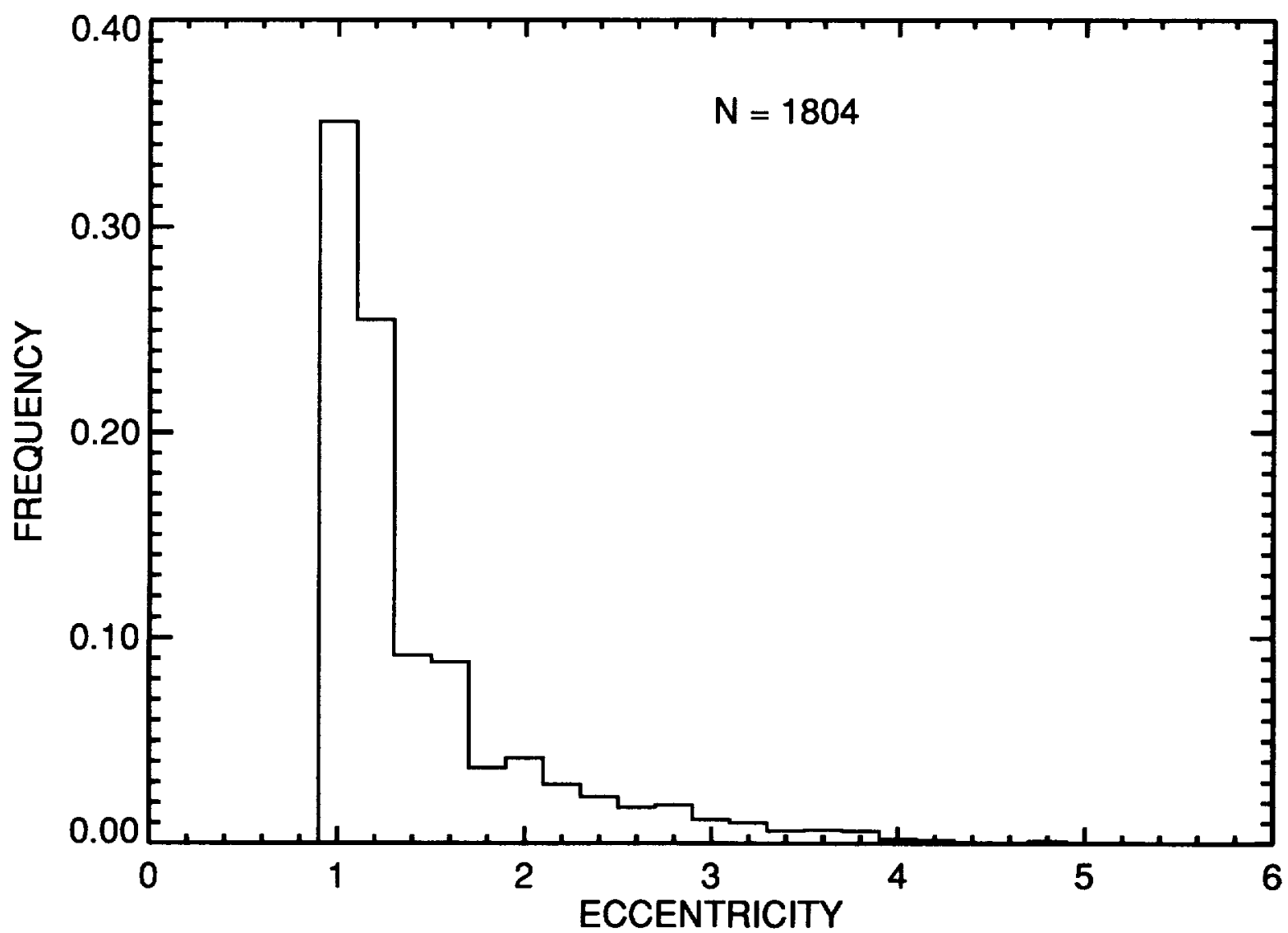

FIG. 7.-Elliptical eccentricity histogram for all 1804 bright points represented in the binary map. The eccentricity is calculated from the best-fit ellipse to each bright point.

FWHM values of these bins are denoted on Figure 9 by the four diamond symbols. The sum of the histograms shown in Figure 10 approximates the data in Figure 8 (referenced to quiet granulation).

The statistical results discussed in this section are summarized in Table 1.

\subsection{Bright-Point Area Distribution}

The association between bright points and magnetic flux sites in the photosphere is well established in a qualitative sense: all bright points occur at sites of magnetic flux, but not all sites of magnetic flux exhibit bright points (Title et al. 1987; Title et al. 1992; Keller 1992; Yi \& Engvold 1993). It has also been found that most of the magnetic field structure in active regions, outside sunspots and pores, exist in small-scale flux tubes with field strengths of 1-2 kG (Stenflo 1973; Tarbell \&

TABLE 1

AR 75811993 SEPTEMBER 20 G-BAND BRIGHT-POINT STATISTICS

\begin{tabular}{|c|c|c|c|c|}
\hline Property & Minimum & Modal & Average & Maximum \\
\hline FWHM diameter $(\mathrm{km}) . . .$. & 120 & 220 & 250 & 600 \\
\hline Aspect ratio $\ldots \ldots \ldots \ldots \ldots$ & 1.0 & 1.0 & 1.5 & 6.5 \\
\hline Area $\left(10^{4} \mathrm{~km}^{2}\right) \ldots \ldots \ldots \ldots$ & 1.0 & 1.1 & 3.1 & $11.0^{*}$ \\
\hline Peak contrast $^{b}(\%) \ldots \ldots \ldots \ldots$ & -19 & 29 & 31 & 75 \\
\hline Mean contrast ${ }^{\circ}(\%) \ldots \ldots \ldots$ & -21 & 26 & 27 & 60 \\
\hline
\end{tabular}

The largest filigree measured has an area of $23.2 \times 10^{4} \mathrm{~km}^{2}$.

belative to quiet-Sun average in image.
Title 1977; Tarbell, Title, \& Schoolman 1979; Stenflo \& Harvey 1985; Keller et al. 1990; Rabin 1992; Solanki 1993 [review]). In view of these facts, the spatial resolution of the September 20 image makes us reasonably confident that the bright points demarcate single, or perhaps tightly clustered, kilogauss flux tubes in the photosphere. It is therefore of interest to attempt to quantify the fraction of the magnetic flux tubes in our image which are demarcated by bright points or other visible proxies such as micropores and pores; i.e., at our spatial resolution of about $0^{\prime \prime} .2$, how much of the magnetic flux in an active region is identified by bright or dark proxy structures? We calculate in this section the fraction of image area occupied by bright points of a given size as well as the area fraction occupied by pores and micropores. Lacking a simultaneous and cospatial magnetogram, we can only compare the resulting values to typical estimates of small-scale flux-tube area fraction (or "filling factor") for comparable active regions. We therefore defer the comparison to the discussion section (\$ 4.2).

As a first-order estimate of the bright-point area fraction, the total area of all objects greater than 5 pixels in area in the bright-point map is divided by the total image area, resulting in a global area fraction of $1.73 \%$. The dominant errors in this calculation are, first, the binary mapping and feature extraction processes, which miss about $15 \%$ of the smallest bright points in the image, and, second, the pixelation of small bright points in the binary map. The magnitude of the first error is estimated (by increasing the number of the smallest bright 


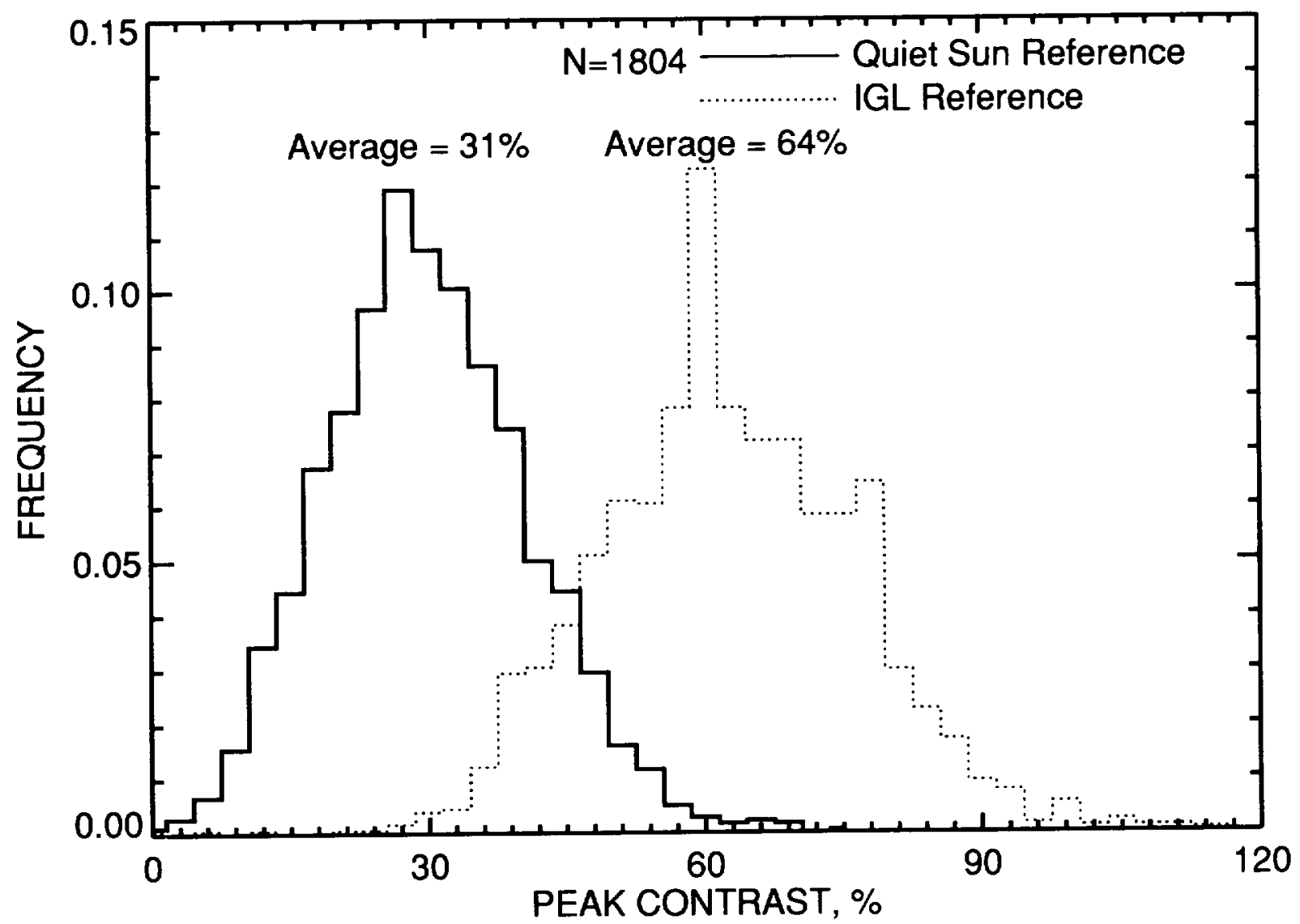

FIG. 8.-Peak-contrast histogram for all 1804 bright points represened in the binary map. Peak contrast is defined as the largest single intensity measured in a

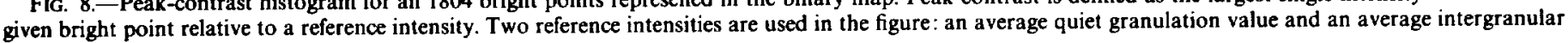
lane value.

points in the binary map by $15 \%$ ) to be on the order of $0.02 \%$ in area fraction. The magnitude of the second error is estimated by calculating the error in the area of a circular bright point represented as a set of square pixels. For the smallest bright points represented in the binary map (circular structures with roughly 3 pixel diameters represented by 5 pixel "crosses") the pixel area underestimates the true circular area by approximately $40 \%$. For larger bright points this error drops rapidly, so that a circular bright point with a diameter of 5 pixels $(0 " 3)$ has an error of roughly $7 \%$. As a first estimate, the effective area of 5 pixel bright points was increased by $40 \%$, resulting in an area fraction correction of $0.05 \%$. Taking both errors into account, the estimated area fraction of all resolved bright points in Figure 2 is $1.80 \%$.

The pore and micropore area fraction is estimated using a binary map created by thresholding the raw image below $-20 \%$ contrast (the average contrast of the larger pores in the image) relative to the image average intensity. A micropore is defined here as any structure between about 0.5 and 1.5 in diameter with an image contrast comparable to that of the larger pores in the image (Spruit \& Zwaan 1981; Topka et al. 1992). The pore map is noise-filtered using an opening operation with a $3 \times 3$ pixel square kernel. The remaining structures in the pore/micropore map occupy approximately $2 \%$ of the total image area. This value is an upper bound because the pore map retains many small objects which may be nonmagnetic noise in the intergranular lane profiles. Adding the bright-point contribution, our estimate of the total area fraction of all magnetic field proxies in the image is $3.8 \%$.

It is of interest to determine the continuous distribution function which describes the number of bright points of a given area in the image. The area fraction as calculated above is given for any discrete area value $A$ by

$$
f_{A}=\frac{A N_{A}}{A_{T}}
$$

where $N_{A}$ is the number of bright points with area $A$, and $A_{T}$ is the total area of the analyzed region. The continuous analog of $N_{A}$ is given by the differential area distribution, $d N / d A$, such that

$$
\left.\frac{d N}{d A}\right|_{A} \Delta A
$$

is the number of bright points in a range of width $\triangle A$ about the area $A$. The differential distribution is found by fitting a continuous function to the discrete histogram of bright-point area.

The bright-point area histogram, corrected for our pixelation and selection effects mentioned above and binned in equal increments of $\ln A$, is shown in $\log -\log$ format in Figure 11 with area expressed in square pixel units. Error bars on the points denote $\pm \sigma$, where $\sigma$ is the standard deviation given by assuming that the number distribution in each bin is Poissonian; i.e., $\sigma_{A}=[N(A)]^{1 / 2}$. Since we find a lognormal dis- 


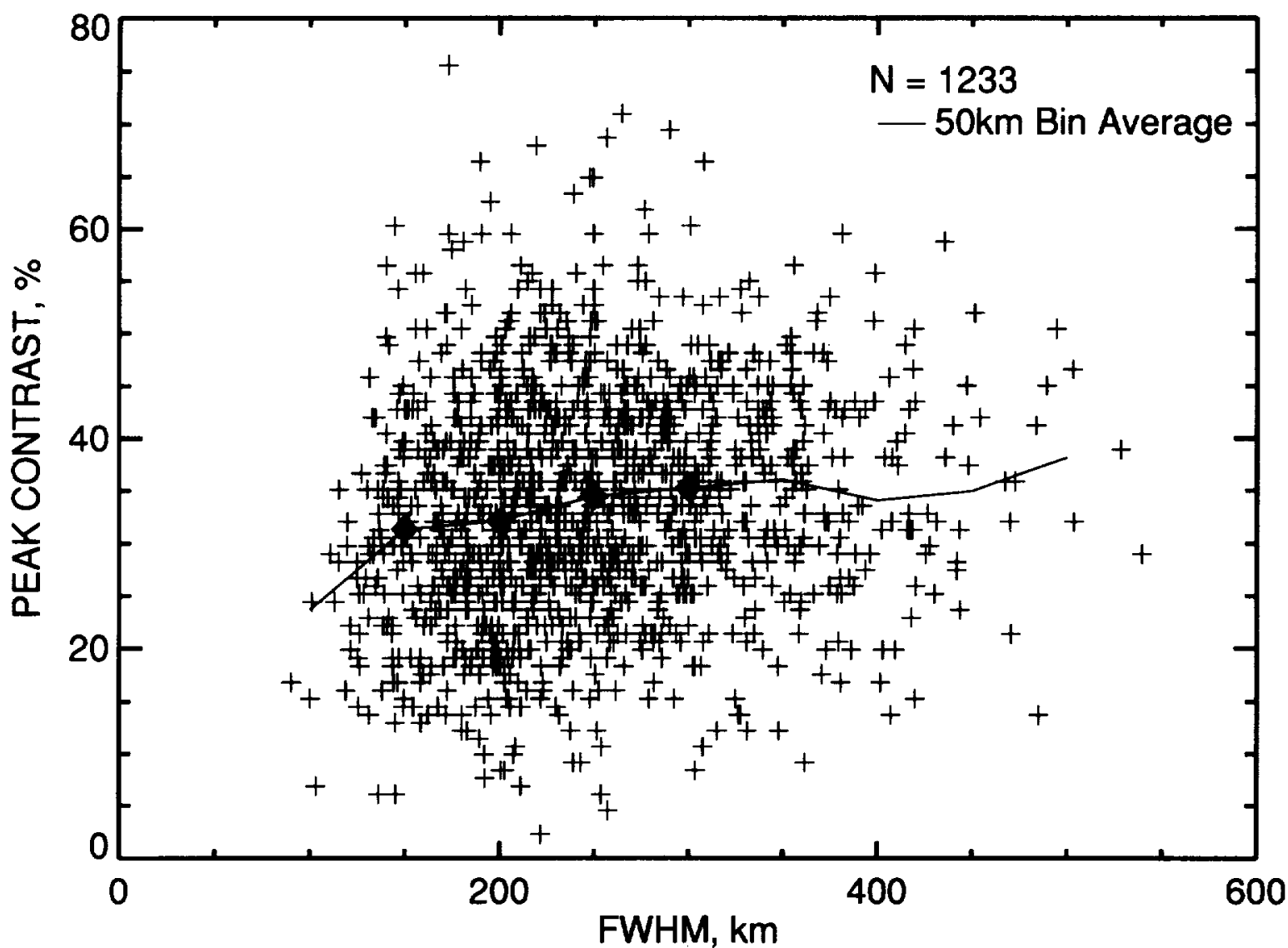

FIG. 9.-Measured peak contrast (relative to quiet Sun) vs. FWHM diameter for 1233 of the bright points shown in Fig. 6 . The solid line plots the $50 \mathrm{~km}$ bin-averaged values of the contrast. Diamond symbols denote the centers of the bins plotted in Fig. 10.

tribution for the FWHM diameter, a related distribution is expected here. Thus we again fit the logarithmically binned histogram with a weighted second-order polynomial as shown on the plot. The form of the differential area distribution as determined by the polynomial fit is then

$$
\frac{d N}{d \ln A}=\frac{d N}{d A} A=e^{-\alpha(\ln A-\mu)^{2}+\gamma},
$$

where $\alpha=1.036, \mu=2.175$, and $\gamma=-0.313$. We note that this distribution includes the filigree in the image. The modal area determined by the distribution is 5.4 pixel $^{2}\left(1.1 \times 10^{4} \mathrm{~km}^{2}\right)$, and the average is $15.1 \mathrm{pixel}^{2}\left(3.1 \times 10^{4} \mathrm{~km}^{2}\right)$. The model area implies a circular bright point with a diameter of $120 \mathrm{~km}$, somewhat less than the model FWHM value determined above, probably because of systematic pixelation and random statistical errors. The average area corresponds to a circular bright point $200 \mathrm{~km}$ in diameter. The total number of bright points calculated by the distribution is found by integrating $d N / d A$ over the range of valid areas for the fit $\left(5-120\right.$ pixel $\left.^{2}\right)$ and normalizing by the number of objects in the binary map (1863, including binary map selection-error correction to the histogram). The number calculated from the distribution is 1888: within $1.3 \%$ of the measured value.

The total area fraction, $f_{T}$, of the bright points is found by calculating the first moment of the differential number distribution and dividing by the image area:

$$
f_{T}=\frac{1}{A_{T}} \int A\left(\frac{d N}{d A}\right) d A \text {. }
$$

When this expression is integrated over the 5-120 pixel $^{2}$ range of the distribution, the resulting bright-point area fraction is $1.83 \%$, in good agreement with the value calculated using the discrete data. Figure 12 shows the bright-point area fraction plotted against area. The solid curve is the fit derived from the lognormal area distribution. The area fraction as a function of bright-point area peaks at about $0.2 \times 10^{5} \mathrm{~km}^{2}$. In Figure 12 the data are binned linearly in area, and as a result the filigree are seen to fall significantly above the area fraction predicted by the fit of Figure 11. For comparison, the only previous small-scale filling factor study we know of is that of Spruit \& Zwaan (1981). Their study of a $\mathbf{M g} b 1$ line-center image in an active region found a bright-point area fraction of $0.8 \%$ and a pore contribution of $3.6 \%$. They find no peak in the brightpoint distribution and in fact show a monotonically increasing filling factor with increasing bright-point size. However, conclusive comparison of their study and our result is difficult owing to differences in bandpass and spatial resolution.

\section{DISCUSSION}

\subsection{Size and Contrast Measurements}

Several previous measurements of photospheric bright-point size agree with the values measured here. However, we find a larger range in bright-point size than has been previously reported. The published data on this subject are sparse, mainly because precise measurements require spatial resolution which is extremely rarely achieved in ground-based observations. Table 2 compares our measured FWHM values and past findings. The only other published distributions of bright-point 
FWHM Bin: 125 to $175 \mathrm{~km}$

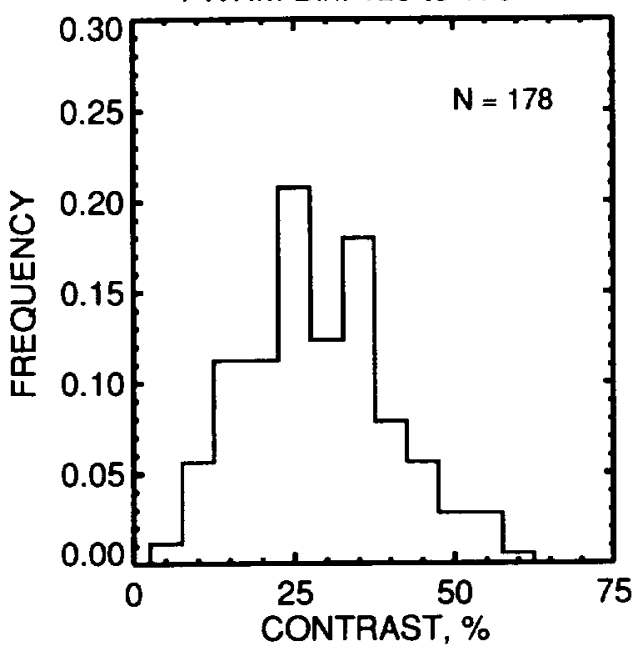

FWHM Bin: 225 to $275 \mathrm{~km}$

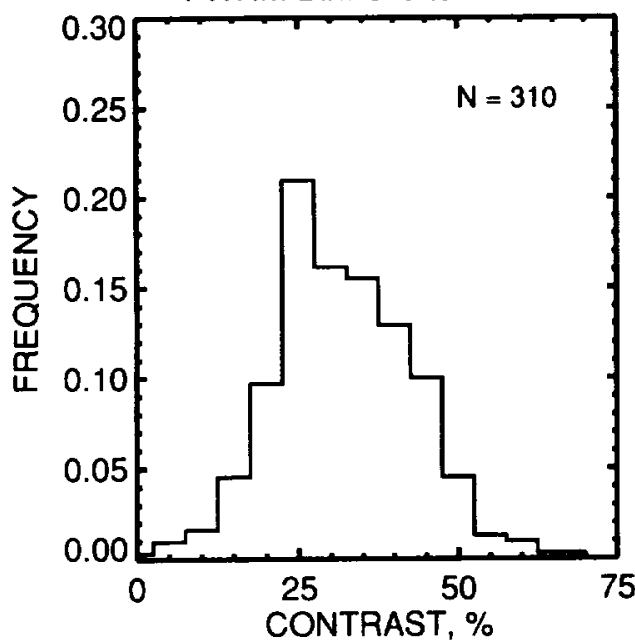

FWHM Bin: 175 to $225 \mathrm{~km}$

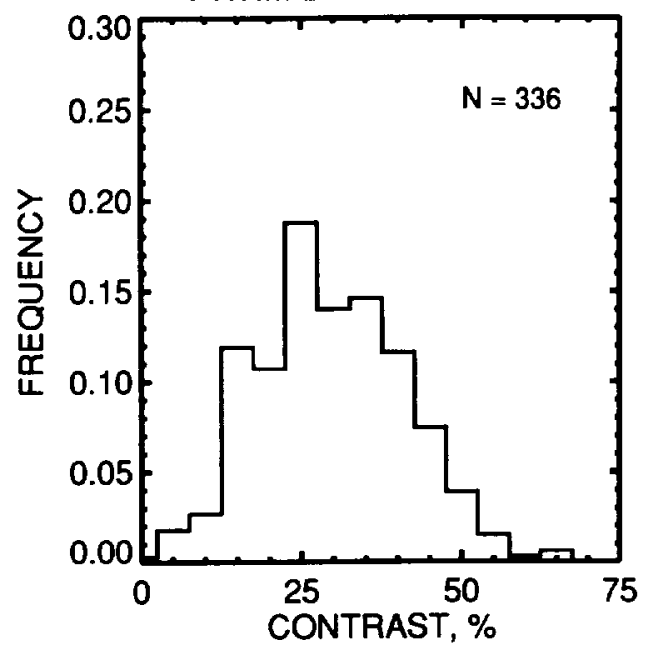

FWHM Bin: 275 to $325 \mathrm{~km}$

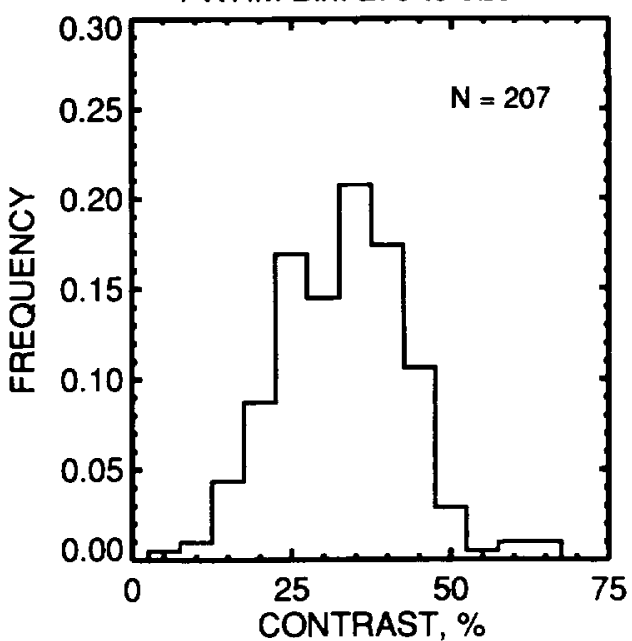

Fig. 10. Contrast histogram for $50 \mathrm{~km}$ size bins created by taking histogram sections centered on the four diamonds plotted in Fig. 9 . The sum of these histograms approximates Fig. 8.

TABLE 2

Bright-Point Size AND Contrast COMParison

\begin{tabular}{|c|c|c|c|c|c|}
\hline Reference & Observatory & $\begin{array}{c}\text { Wavelength } \\
(\AA)\end{array}$ & $\begin{array}{c}\text { Bandpass } \\
\text { (A) }\end{array}$ & $\begin{array}{c}\text { Size Range } \\
{[\mathrm{km}(\operatorname{arcsec})]}\end{array}$ & $\begin{array}{c}\text { Uncorrected } \\
\text { Contrast Range } \\
(\%)\end{array}$ \\
\hline This study .......... & La Palma & (G band) 4305 & 12 & $120-600(0.17-0.83)$ & $-19-75$ \\
\hline Mehltretter $1974 \ldots$ & Sacramento Peak & (Ca II k) 3934 & 16 & $180-225(0.25-0.31)$ & $23-30$ \\
\hline Koutchmy $1978 \ldots \ldots \ldots \ldots$ & Sacramento Peak & 6441 & 0.5 & NA & $20^{b}$ \\
\hline Spruit \& Zwaan 1981 ...... & Sacramento Peak & $(\mathrm{Mg} b 1) 5183$ & 0.17 & $220-435(0.30-0.60)$ & $5-60$ \\
\hline Muller \& Keil 1983 ......... & Pic-du-Midi & 5750 & 60 & $145-435(0.20-0.60)$ & $-3-18$ \\
\hline Muller \& Auffret 1991 ...... & Pic-du-Midi & 5750 & 60 & $145-435(0.20-0.60)$ & $-5-25$ \\
\hline de Boer \& Kneer $1992 \ldots$. & La Palma & 5500 & 100 & $145-300^{c}(0.20-0.41)$ & NA \\
\hline Yi \& Engvold $1993 \ldots \ldots \ldots$ & La Palma & $(H x) 6564$ & 0.1 & $290-600(0.40-0.83)$ & NA \\
\hline Von der Lühe $1994 \ldots \ldots \ldots$ & Sacramento Peak & 5850 & 80 & $120-500^{c}(0.17-0.67)$ & $\mathbf{N A}$ \\
\hline
\end{tabular}

- Relative to quiet-Sun average in image

b Single-point study.

c Speckle interferometrically corrected. 


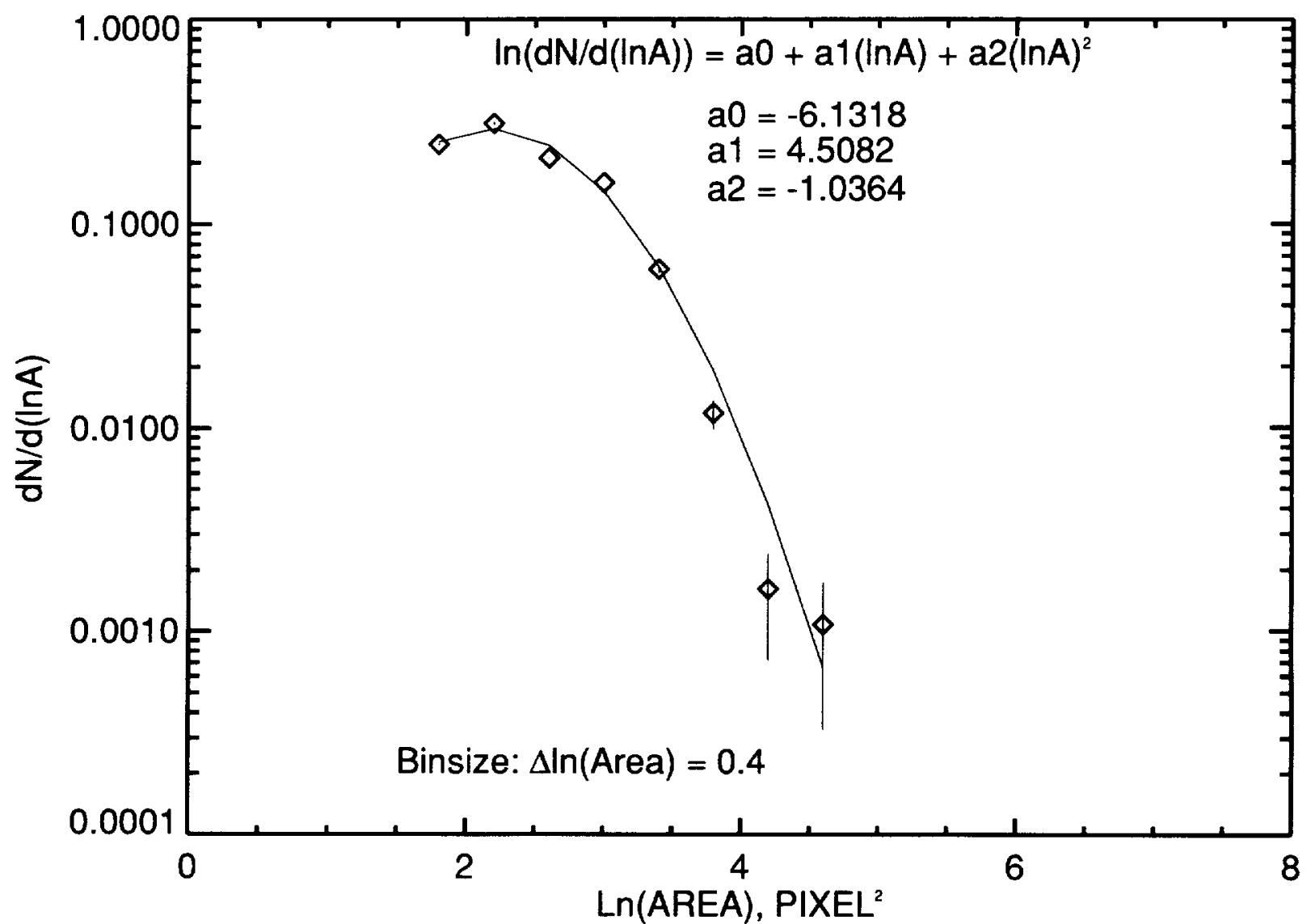

Fig. 11.- Number of bright points per unit area as a function of bright-point area for all 1804 bright points extracted from Fig. 2. The solid line is a least-squares second-order polynomial fit to the data in the area range 5-120 pixels ${ }^{2}\left(0.1 \times 10^{5}-2.5 \times 10^{5} \mathrm{~km}^{2}\right)$. The error bars denote the standard deviation of a Poisson distribution for the number of bright points in a given area bin.

size (Muller \& Keil 1983; Muller \& Auffret 1991) are derived from quiet-Sun network observations at $5750 \AA$. These distributions show a modal size of $240 \mathrm{~km}\left(0^{\prime \prime} .3\right)$ and a maximum bright-point size of $450 \mathrm{~km}\left(0^{\prime \prime} 6\right)$. Assuming that the modal size measurement is point-spread function (PSF) limited, we can correct for the wavelength difference between our data and the Pic du Midi data by multiplying their modal value by the ratio $4300 / 5750$. The correction results in a "G-band equivalent" modal value for the Pic du Midi distributions of $180 \mathrm{~km}$, somewhat less than our measurements of bright points within active region plage. Since the maximum bright-point size measurement is most likely not PSF limited, the wavelength correction cannot be applied: we measure significantly larger bright points than are found in the network. Taking the region of Figure 2 as typical, this suggests that plage regions in general have a larger bright-point size range compared to the network.

Comparing the bright-point contrast values measured here to previous studies is complicated by the fact that many authors prefer to quote "corrected" rather than observed contrast values. The correction is often based on the assumption that the effect of atmospheric and telescopic blur can be modeled as a Gaussian profile which adds in quadrature with the intrinsic Gaussian profile of the bright points (e.g., Mehltretter 1974; Spruit \& Zwaan 1981; Muller \& Keil 1983). Since the observed profile of the bright points we measure is not purely Gaussian (see Fig. 5), we have not attempted to correct our contrast values in this manner. Other authors have used optimal filtering (Koutchmy 1978) or speckle interferometric methods (de Boer \& Kneer 1992; Von der Lühe 1994) to arrive at corrected contrast values. It is therefore probably most meaningful to compare uncorrected contrast values under the assumption that seeing effects are probably minimal if 0.25 bright points can be seen in the raw data at all. Table 2 compiles the uncorrected contrast values from this and earlier studies. The G-band observations are seen to give contrast values about a factor of 2 higher than those measured in the continuum; only the line-center observations of Spruit \& Zwaan (1981) approach the maximum values we measure.

Interpretation of the contrast comparisons shown in Table 2 is complicated by the fact that observations in different bandpasses will in general refer to different temperature regimes and by inference, different heights in the solar atmosphere. Thus observations in temperature-sensitive molecular bands such as the $\mathrm{G}$ band are attributed to the "upper photospheric" layers of the atmosphere, while continuum observations generally sample the "lower photospheric" layers. Since magnetic flux tubes may "fan out," or diverge rapidly in diameter, with increasing height in the atmosphere, contrast measurements in different wavelengths regimes may refer to significantly different topologies of the magnetic field.

The relation of G-band bright-point size to peak contrast is particularly difficult to interpret in light of currently accepted small-scale magnetic flux-tube models. Specifically, the modal size of $220 \mathrm{~km}$ established in Figure 6 is consistent with, or 


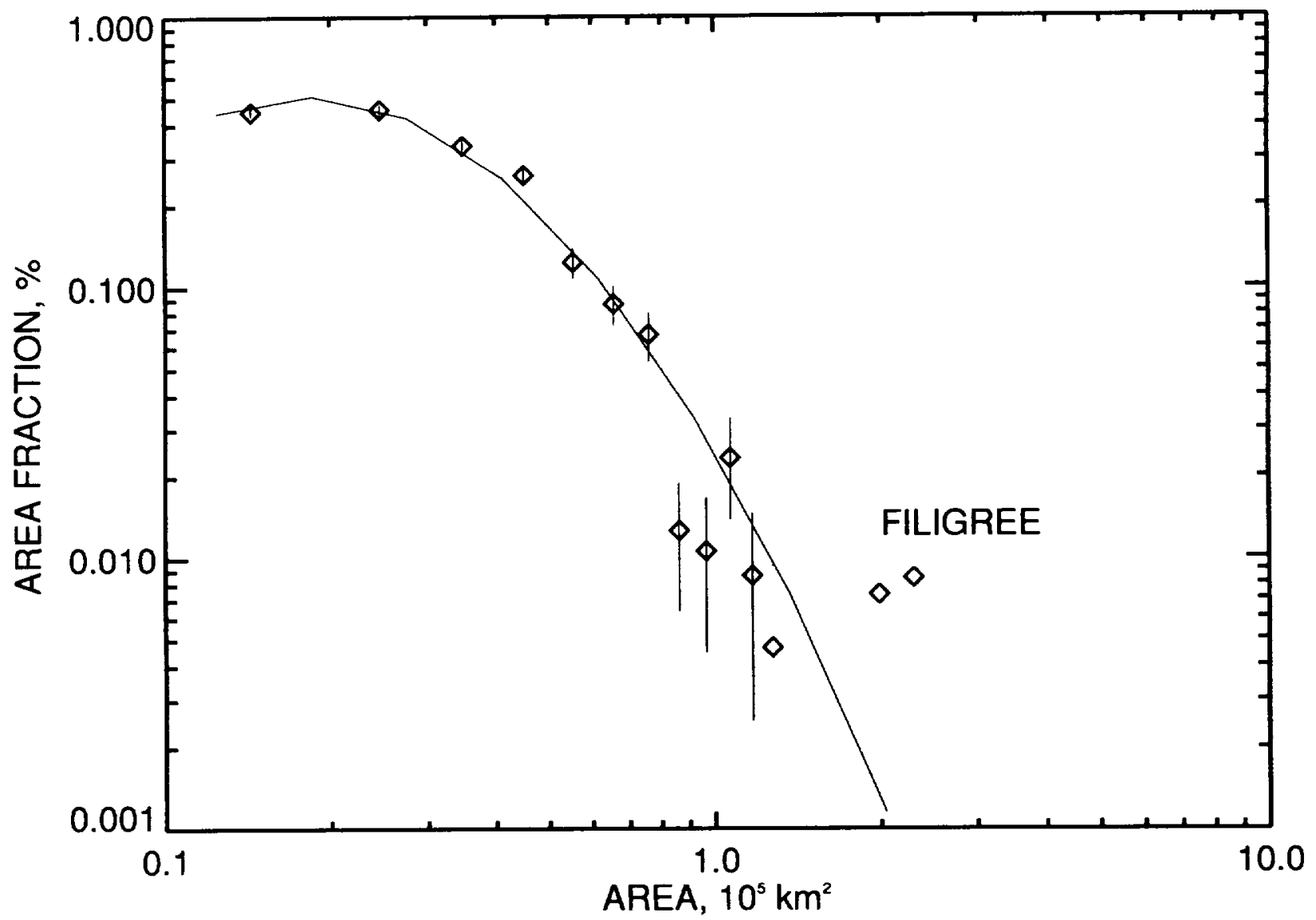

FIG. 12.-Logarithm of bright-point area fraction vs. bright-point area. The solid line is derived from the fit shown in Fig. 11. The error bars denote the standard deviation of a Poisson distribution for the number of bright points in a given area bin.

even below, the predictions of small-scale flux-tube size from detailed MHD numerical simulations which refer to the lower photosphere (Spruit 1976; Deinzer et al. 1984; Knölker \& Schüssler 1988; Grossman-Doerth et al. 1994). Based on the mechanism of radiative diffusion from the surrounding environment into the relatively evacuated flux tubes (the "hotwall" model), these same models predict that the bright points associated with the flux tubes display a definite trend with size: flux tubes larger than about $300 \mathrm{~km}$ become darker with increasing size until at diameters of about $400 \mathrm{~km}$ the tubes are predicted to have negligible contrast. Further, owing to a depression on the order of $100 \mathrm{~km}$ of the isotherms within the flux-tube environment (Amer \& Kneer 1993), the radiative diffusion originates at a level significantly below the external continuum formation $\left(\tau_{5000}=1\right)$ level. Keller (1992), Topka et al. (1992), and Von der Lühe (1994) have published observations which largely substantiate these predictions in continuum wavelengths.

However, it is clear from Figure 1 that the G-band structures are not depressed within the granulation but appear vertically extended above it. In addition, we measure bright points up to $500 \mathrm{~km}$ in diameter with contrast values equal to or greater than those measured for much smaller bright points. That the larger structures may be unresolved groups of "hot-wall " illuminated flux tubes seems unlikely, since the hot-wall mechanism apparently fails for a proximate group of (cool, evacuated) flux tubes. In short, there are strong indications that the hot-wall model fails to apply to G-band bright points.
An alternative mechanism presently under investigation is collisional excitation of $\mathrm{CH}$ to the $A^{2} \Delta$ electronic state by fieldaligned currents within the flux tubes followed by $G$-band radiation to the ground state - a process analogous to the terrestrial auroral phenomena.

Note that Figures 8 and 10 suggest a constraint on any mechanism proposed for the G-band brightness source: the measured contrast distribution, irrespective of size, is fairly symmetric around a well-defined mean value. The low skew and the relatively constant width of the distributions shown in Figure 10 imply that bright points maintain a relatively constant characteristic brightness throughout their lifetime. For instance, if the G-band source function were driven by impulsive heating followed by exponential decay, the distributions would be positively skewed, since the most probable contrast for any given bright point would be significantly lower than the peak contrast attained during the heating. Since the estimated bright-point lifetime is on the order of 10-20 minutes (Muller 1994), the mechanism(s) responsible for bright-point intensity must maintain their energy input at fairly constant levels over this timescale.

\subsection{Area Distribution and the Relation to the Magnetic Filling Factor}

There are several observations of magnetic filling factor in active regions outside sunspots with which our measurements of proxy area fraction can be compared. Tarbell et al. (1979) found an $8.5 \%$ strong-field filling factor in disk-center plage, 
assuming a field strength at the height of line formation (mid photosphere) of $1200 \mathrm{G}$. This corresponds to a mean measured longitudinal field strength of about $100 \mathrm{G}$. Schrijver (1987) and Schrijver \& Harvey (1994) generalized this result, showing that solar plage regions exhibit a fairly constant mean field value of 135-150 G across a wide set of region topology and age variations. Considering a slightly higher field strength at the continuum formation height, say $1500 \mathrm{G}$, this implies a filling factor of $9 \%-10 \%$. Both Strous (1994b) and Topka (1995) estimate a $20 \%$ filling factor from high-resolution magnetograms in a plage region made with the SOUP filter (Title et al. 1989) at La Palma. Filling factors of between $15 \%$ and $25 \%$ are also estimated in the low spatial resolution observations of plage regions at disk center by Solanki \& Brigljevic (1992); these estimates are derived from $10^{\prime \prime}$ resolution observations which may have been centered on a stronger than average concentration of flux tubes. With the possible exception of the Solanki \& Brigljevic (1992) values (derived from very low noise data), all of the filling factor values are sensitive to the threshold used to define the active region extent, which is in turn dependent on magnetogram noise levels. Nevertheless, it is probably reasonable to infer that magnetic filling factors on the order of $10 \%$ or larger in the photosphere are typical for active region plage.

Given the established correlation of bright points and pores with small-scale magnetic flux tubes, we can infer a "proxy" magnetic filling factor in Figure 2 from the area measurements of $\S 3.3$. Assuming that the entire image area contains plage, the implied filling factor is equal to the measured area fraction of bright points and pores $(3.8 \%)$. The assumption that the plage occupies the entire image area is difficult to verify. In fact, there are regions on the image which appear to be simply quiet granulation and which may be relatively field-free. However, a comparison of Figure 2 with a Kitt Peak magnetogram taken roughly $2 \mathrm{hr}$ later with 2 " spatial resolution (at best) shows that the majority of the image area registers at least some flux. Nevertheless, if we assume that the quiet granulation areas are not magnetic, and subtract their area from the reference area used to determine the filling factor, the implied filling factor increases only slightly to $4.3 \%$.

Since the area under analysis is by all appearances a typical active region plage, we conclude that the implied filling is at least a factor of 2 below the expected magnetic filling factor. This implies that over $50 \%$ of the small-scale magnetic flux tubes permeating the area of Figure 2 are not demarcated by any visible proxy; i.e., they exhibit zero contrast with respect to their immediate surroundings. The following three scenarios seem plausible as explanations for the lack of proxy structures on such a large fraction of the magnetic flux:

1. The bright points and micropores are cospatial with the underlying magnetic flux tubes but are significantly smaller in area.

2. There exists a profusion of subresolution flux tubes which possess intrinsic brightness values comparable to those of the larger bright flux tubes but at a resolution of 0.2 are spatially averaged into low-contrast structures.

3. The observed and/or intrinsic brightness of flux tubes is sporadic; e.g., there may exist numerous flux tubes of resolvable size in the image which temporarily (or perhaps permanently) are not demarcated by bright points.

The first scenario is supported by comparisons of highresolution magnetograms and filtergrams (Simon \& Zirker 1974; Title et al. 1987, 1992; Keller 1992; Yi \& Engvold 1993) which show that bright points, while always cospatial with flux tubes, are frequently smaller. Simon \& Zirker (1974), comparing magnetograms in the $\mathrm{Fe} I 6302 \AA$ line with $6303 \AA$ continuum images, found the magnetic FWHM diameter in an active region to be an average of 1.3 times larger than the FWHM of the associated $6303 \AA$ feature. This results in an average area ratio of 1.7 , which, when applied to our measured filling factor, increases it to about $8 \%$, approaching the value of the "expected" filling factor. Title et al. (1995) show that an isolated bright points embedded in a typical intergranular lane can be modeled by the sum of two Gaussian profiles, one with positive amplitude and one with negative amplitude. The resulting FWHM of the model bright point is at most a factor of 1.4 less than the FWHM value of the positive Gaussian profile, which, for instance, would describe an isolated feature on a magnetogram. This results in the magnetogram feature having an area twice as large as the associated bright point, roughly in agreement with the findings of Simon \& Zirker (1974).

The existence of subresolution bright points is suggested by the fact that our measured FWHM histogram peaks near the diffraction-limited FWHM of the SVST at $4300 \AA$ (shown by the dashed line in Figure 6), which is perhaps indicative of a continuing distribution to smaller sizes. In addition, detailed investigation of Figures 2 and 3 shows that there are many low-contrast amorphous features among dense concentrations of bright points or around pores. Both their contrast and their general shape match what we would expect from a group of unresolved bright points. For example, the amorphous region at $[8.5,9.0]$ in Figure 3 is most likely such a group. We note that the bright-point binary mask as shown in Figure 4 registered several objects on the periphery of this region but none in the interior. If the unresolved bright points follow the area distribution measured for larger bright points, their total contribution to the filling factor is expected to be small, since the number distribution declines in the unresolved range. We can estimate this contribution by integrating the filling factor expression of equation (6) from an area of 0.001 pixel $^{2}\left(2 \mathrm{~km}^{2}\right.$, a "near-zero" value) to 5 pixel $^{2}\left(1.1 \times 10^{4} \mathrm{~km}^{2}\right.$, the lower limit of the measured data). The integrated filling factor is about $0.1 \%$, which indicates that an unresolved bright-point contribution to the filling factor is expected to be insignificant if the ultra-small-scale bright points follow the distribution measured for the larger points. Conversely, if the number of bright points were to increase rapidly below the resolution of our observations, it is conceivable that their contribution to the area fraction could become significant.

This discussion assumes that there is no size cutoff below which flux tubes no longer exist in the photosphere. Theoretical studies have addressed the issue of a lower limit to flux-tube size in the photosphere through various models. The convective collapse mechanism of flux-tube formation estimates a lower limit equal to the thermal boundary-layer width, which is calculated to be only a few kilometers in the lower photosphere (Schüssler 1987). However, Venkatakrishnan (1986) argues that radiative pressure stops the convective collapse at a diameter of about $100 \mathrm{~km}$. Semiempirical models which require specific opacity ratios between the flux tube and its surroundings (Solanki 1986) also place the lower limit on fluxtube diameter at about $100 \mathrm{~km}$. Studies of flux-tube interaction with acoustic waves (Ryutova 1986; Ryutova, Tajima, \& Kaisig 1995) indicate that larger flux tubes are split into successively smaller ones with the lower diameter limit lying 
between 40 and $60 \mathrm{~km}$ (Ryutova 1995). The general consensus seems to be that the minimum flux-tube diameter is somewhat, though not very much, below our resolution limit, leaving the possibility for a large "unresolved" contribution to the filling factor uncertain.

The third scenario is supported again by the high-resolution magnetogram/bright-point observations (Simon \& Zirker 1974; Title et al. 1987, 1992; Keller 1992; Yi \& Engvold 1993), which show that there are flux tubes (or perhaps unresolved clusters of tubes) in magnetograms with no associated positive or negative contrast structures in simultaneous visible images. Other elements identical in size and shape to these flux tubes show corresponding bright points or micropores in line-center or continuum images. Simon \& Zirker (1974) found that out of 15 identified flux structures in an active region, four showed no associated dark or bright structures in the continuum. Although the process is clearly lacking in statistical rigor, we can apply this ratio of invisible flux to our data to arrive at a corrected filling factor for our image of $5 \%$ - a small but significant increase.

This scenario raises the question of what causes some flux tubes to be bright in an image while others of seemingly identical structure are dark. Dynamic simulations of small flux tubes in granulation (Steiner, Knölker, \& Schüssler 1994) have shown that small flux tubes may undergo large "bending" oscillations as a consequence of their interaction with the convective flow field, resulting in large temporal fluctuations in the brightness observed in any given direction at a given time. Other possibilities for intermittent flux-tube illumination include acoustic (Kalkofen 1990), torsional, or Alfvén wave excitation of the flux-tube interior gas. The auroral mechanism mentioned in the previous section is also capable of producing the effect, given an intermittency of the currents within the flux tubes.

Of course, there is the possibility that the filling factor discrepancy is explained by a combination of the three scenarios. In fact, this seems likely, since each of the scenarios can be supported by observations and/or theory, while none of them is completely refuted. Obviously a cotemporal magnetogram at approximately the same spatial scale as the G-band observations would be very useful for further investigating these issues. To this end, we obtained cotemporal and cospatial magnetograms as well as continuum, $\mathrm{H} \alpha$, and $\mathrm{Ca}$ II $\mathrm{K}$-line filtergrams using the SOUP filter on a second beam line at the SVST in the summer of 1994. Analysis of these images is in progress. Preliminary results indicate that the SOUP magnetograms achieve, in the best seeing conditions, spatial resolution on the order of 0 " $4-0$ ".5, which should be sufficient to make useful conclusions on the physical relation of G-band bright points to the small-scale magnetic field in the photosphere. More important, the data obtained in 1994 consist of time series of multiwavelength observations up to $4 \mathrm{hr}$ in length with typical time between frames of $20 \mathrm{~s}$. This will allow temporal as well as spatial correlations of bright points with magnetic field to be established on scales below 0.5 .

This work was supported by NASA contracts NAS5-30386 at Stanford and NAS8-39747 at LPARL. NSO/Kitt Peak data used here are produced cooperatively by NSF/NOAO, NASA/ GSFC, and NOAA/SEL. The SVST is operated by the Swedish Royal Academy of Sciences at the Spanish Observatorio del Roque de los Muchachos of the Instituto de Astrofisica de Canarias. We thank Mary Barreto of IAC and Rolf Kevar, Paco Armes, and Göran Hosinsky of the SVST staff for support and assistance during these and the summer 1994 observations. Bob Noyes, George Simon, Ken Topka, and Rob Rutten provided many helpful comments and suggestions during the course of this project. Ken Topka also wrote the original version of the nearest-neighbor feature extraction routine. We thank Bill Rosenberg (LPARL) for providing the ellipse-fitting routine. Special thanks to Zoe Frank at LPARL for providing crucial computing support and advice. We thank the referee for a thorough and expedient reading as well as several important clarifications. T. E. B. thanks Phil Scherrer, Margie Stehle, Anne, and Maddie for support and encouragement.

\section{REFERENCES}

Amer, M A \& Kneer, F. 1993, A\&A, 273, 204

Bogdan, T. J., Gilman, P. A., Lerche, I., \& Howard, R. 1988, ApJ, 327, 451 Chapman, G. A. 1970, Sol. Phys., 13, 78

de Boer, C. R., \& Kneer, F. 1992, A\&A, 264, L24

Deinzer W Hensler G Schüssler. M. \& Weisshaar, E. 1984, A\&A, 139, 435

Dunn, R. Mann G R \& Simon, G W. 1973, BAAS, 4, 381

Dunn, R. B., \& Zirker, J. B. 1973, Sol. Phys., 33, 281

Foukal, P., Duvall, T., Jr., \& Gillespie, B. 1981, ApJ, 249, 394

Foukal, P., \& Fowler, L. 1984, ApJ, 281, 442

Grossman-Doerth, U Knölker, M. Schüssler, M., \& Solanki, S. K. 1994, A\&A, 285, 648

Haralick, R. M., Sternberg, S. R., \& Zhuang, X. 1987, IEEE Trans., PAMI-9 (No. 4), 532

Hirayama, T. Hamana, S. \& Mizugaki, K. 1985, Sol. Phys., 99, 43

Kalk W 1990 in IAU Symp 138, Solar Photosphere: Structure, Convection, and Magnetic Fields, ed. J. O. Stenflo (Dordrecht: Kluwer), 185

tion, and Magnetic Fields, ed. J. O
Keller, C. U. 1992, Nature, 359, 307

Keller, C. U., Solanki, S. K., Tarbell, T. D., Title, A. M., \& Stenflo, J. O. 1990, A\&A, 236, 250

Knölker, M \& Schüssler, M. 1988, A\&A, 202, 275

Koutchmy, S. 1978, A\&A, 61, 397

Mehltretter, J. P. 1974, Sol. Phys., 38, 43

Muller, R. 1994, in NATO ASI Ser. 433, Solar Surface Magnetism, ed. R. J. Rutten \& C. J. Schrijver (Dordrecht: Kluwer), 73

Muller, R \& Auffret, H, 1991, A\&A, 246, 264

Muller, R., \& Auffret, H, 1991, A\&A, 246, 264 113

Muller, R., \& Keil, S. L. 1983, Sol. Phys., 85, 113

Muller, R., \& Roudier, Th. 1984, Sol. Phys., 94, 33

Muler, Roudier, Th Vigneau, J \& Auffret. H. 1994, A\&A, 283, 232

Parker E 1990 in Mechanisms of Chromospheric and Coronal Heating, ed. P. Ulmschneider, E. R. Priest, \& R. Rosner (Berlin: Springer), 615
Rabin, D. 1992, ApJ, 390, L103

Ramsey, H. E.Schoolman, S A.\& Title, A. M. 1977, ApJ, 215, L41

Ryutova, M. P. 1986, in Proc. Joint Varenna-Abastumani Int. School on Plasma Astrophysics (Paris: ESA), 71 1995, private communication

Ryutova, M. P., Tajima, T. \& Kaisig, M. 1995, in preparation

Sctellar Granulation, ed. R. J. Rutten \& G. Severino (Dordrecht: Kluwer), 161

ed. R. J. Rutten \& G. Severino(Dordrech L. \& Rehn, J. 1985, Appl. Optics, 24 (No. 16), 2558

Schrijuer, C. J. 1987, A\&A, 180, 241

Schrijuer C. J \& Harvey, K. L. 1994, Sol. Phys., 150,

Schüssler M 1987 in The Role of Fine-Scale Magnetic Fields in the Solar

Atmosphere, ed. E.-H. Schröter, M. Vázquez, \& A. A. Wyller (London: Cambridge Univ. Press), 223

Sheeley, N. R. 1969, Sol. Phys., 2, 347

1992 in ASP Conf Ser. 27, The Solar Cycle, ed. K. L. Harvey (San Francisco: ASP), 1

Simon, G. W., Brandt, P. N., November, L. J., Scharmer, G. B., \& Shine, R. A 1994, in NATO ASI Ser. 433, Solar Surface Magnetism, ed. R. J. Rutten \& C J Schrijver (Dordrecht: Kluwer), 261

Simon G. W. \& Zirker, J. B. 1974, Sol. Phys., 35, 331

Solanki, S. K. 1986, A\&A, 168, 311 1993, Space Sci. Rev., 63

Solanki, S. K., \& Brigljevic, V. 1992, A\&A, 262, L29

Spruit, H. C. 1976, Sol. Phys. 50, 269

Spruit, H \& Zwaan C. 1981. Sol. Phys., 70, 207

Spruit, H. C. \& Z Zwer, M., \& Schüssler, M. 1994, in NATO ASI Ser. 433, Solar

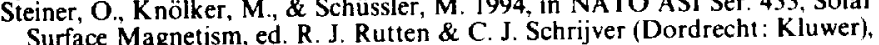
73 
Stenflo, J. O. 1973, Sol. Phys., 32, 41

Stenflo, J. O., \& Harvey, J. W. 1985, Sol. Phys. 95,99

Strous, L. 1994a, in NATO ASI Ser. 433, Solar Surface Magnetism, ed. R. J. Rutten \& C. J. Schrijver (Dordrecht: Kluwer), 73 1994b, Ph.D. thesis, Univ. Utrecht

Tarbeli, T.D., \& Title, A. M. 1977, Sol. Phys., 52, 13

Tarbell, T. D., Title, A. M., \& Schoolman, S. A. 1979, ApJ, 229, 387

Title, A. M., Berger, T. E., Tarbell, T. D., Shine, R. S., \& Scharmer, G. B. 1995, in preparation

Titie, A. M., Tarbell, T. D., \& Topka, K. P. 1987, ApJ, 317, 892

Title, A. M., Tarbell, T. D., Topka, K. P., Shine, R. A., Simon, G. W., Zirin, H., $\&$ the SOUP Team. 1989, ApJ, 336, 475
Title, A. M., Topka, K. P., Tarbell, T. D., Schmidt, W., Balke, C., \& Scharmer, G. $1992, \mathrm{ApJ}, 393,782$

Tomita, F. 1990, Computer Analysis of Visual Textures (1st ed.; Boston: Kluwer)

Topka, K.P. 1995, private communication

Topka, K. P., Tarbell, T. D., \& Title, A. M. 1992, ApJ, 396, 351

Van Ballegooijen, A. A. 1986, ApJ, 311, 1001

Venkatakrishnan, P. 1986, Nature, 322, 156

Von der Lühe, O. 1994, A\&A, 281,889

Wang, Y. -M., Nash, A. G., \& Sheeley, N. R., Jr. 1989, Science, 245, 712

Yi, Z., \& Engvold, O. 1993, Sol. Phys., 144, 14 


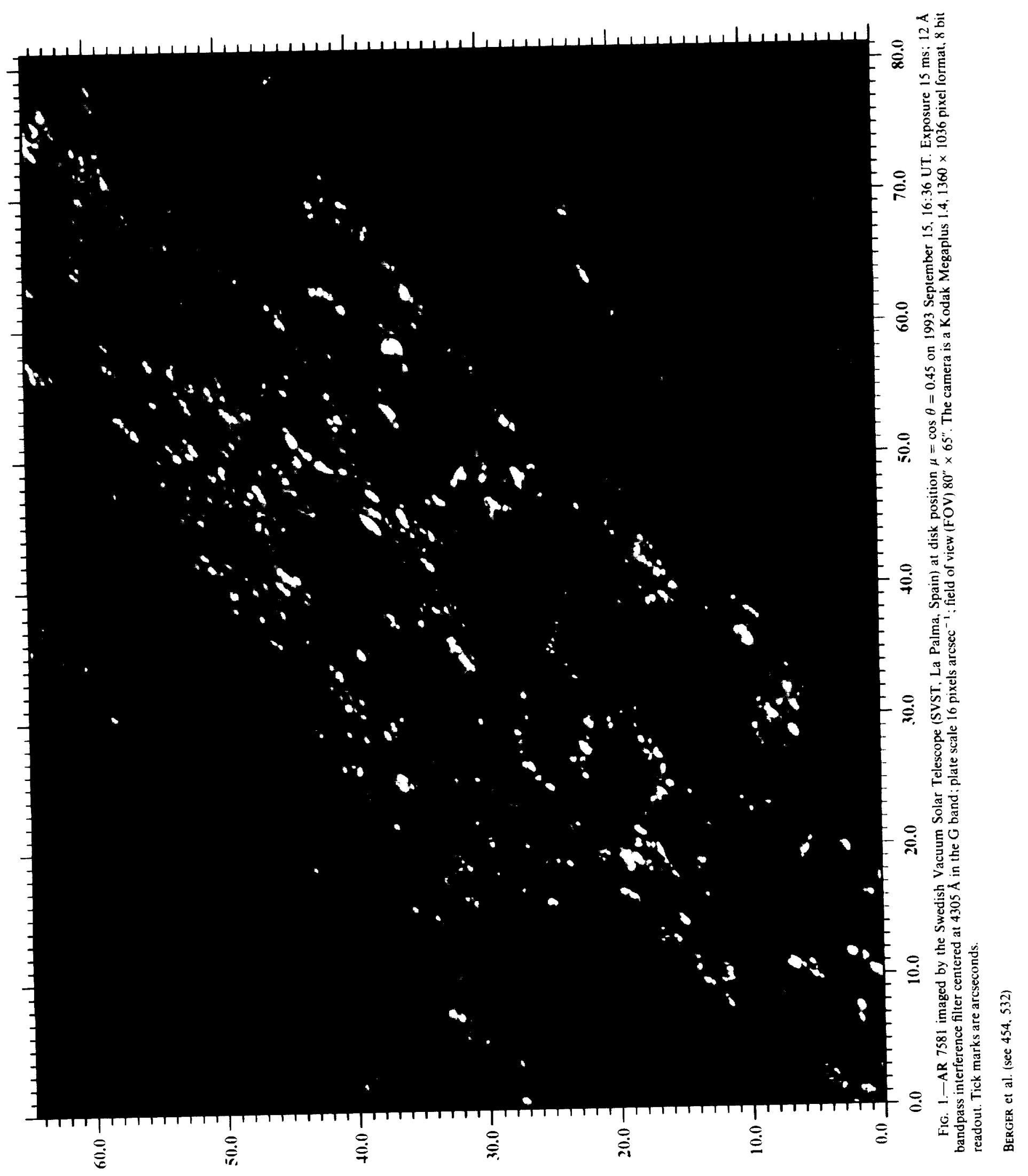




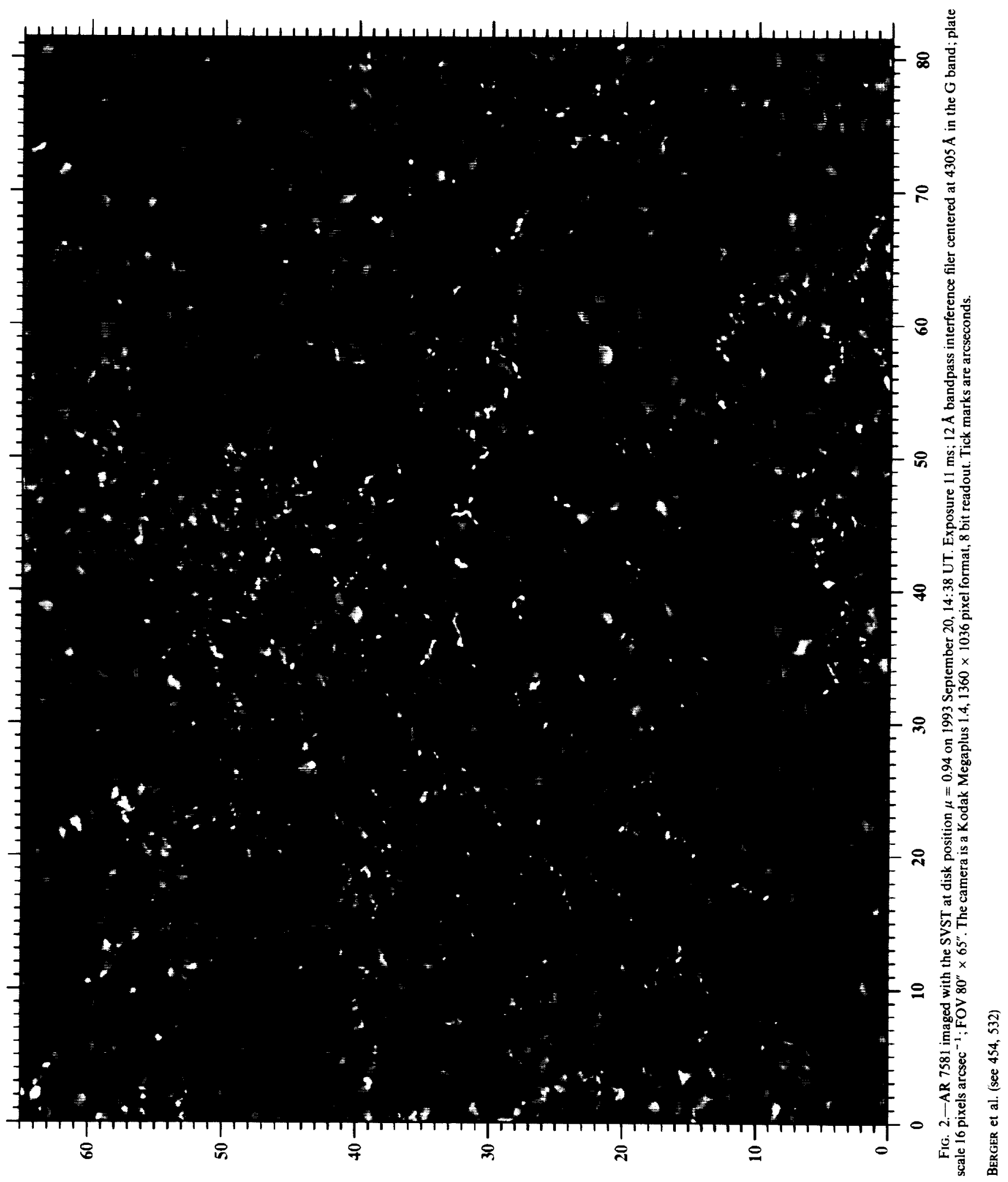




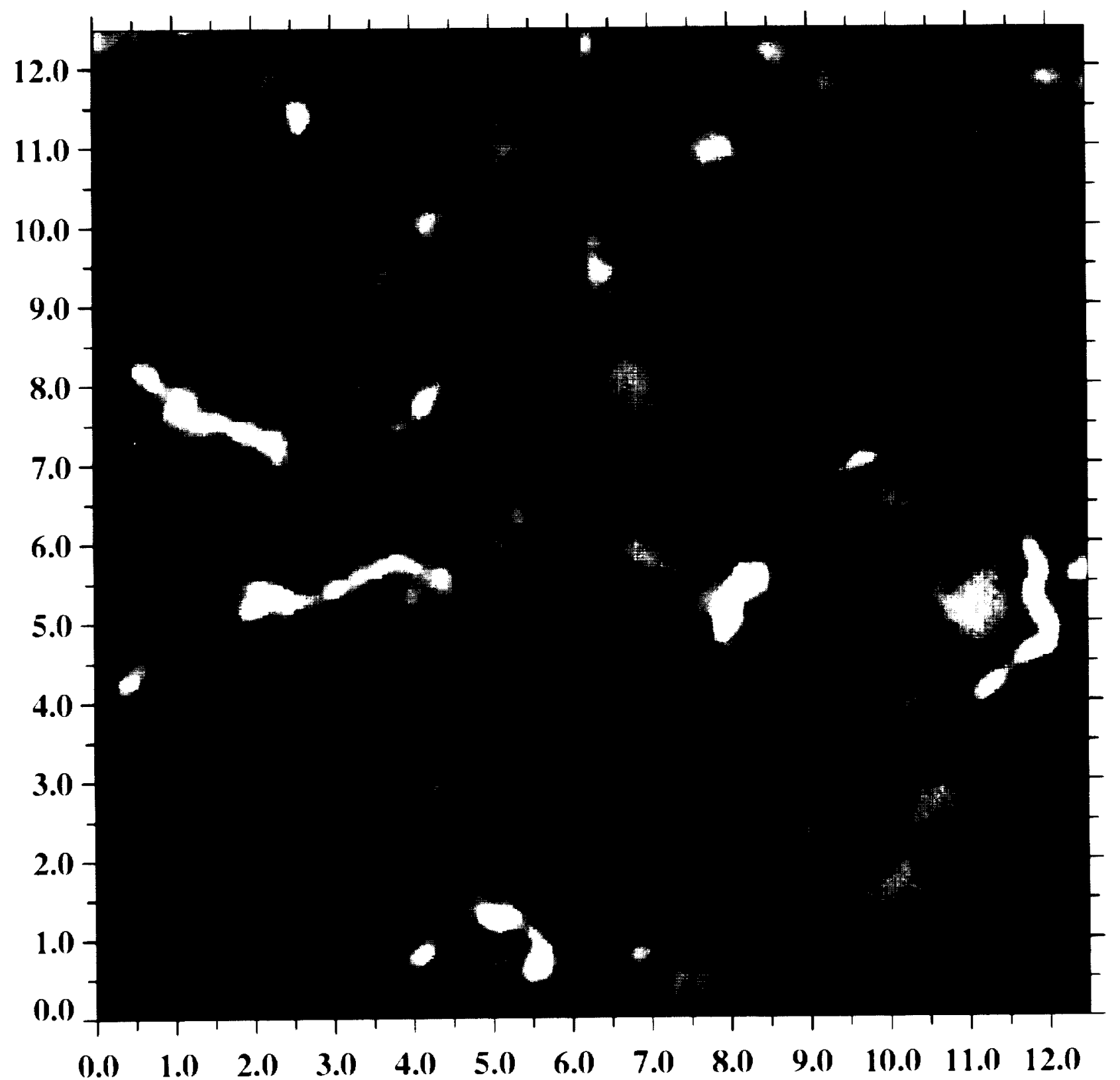

FIG. 3.-Central $200 \times 200$ pixel area of Fig. 2 magnified by a factor of 3. Tick marks are 0.5 . The area shows the full range of bright-point size and shape, including the filigree.

Berger et al. (see 454, 532) 


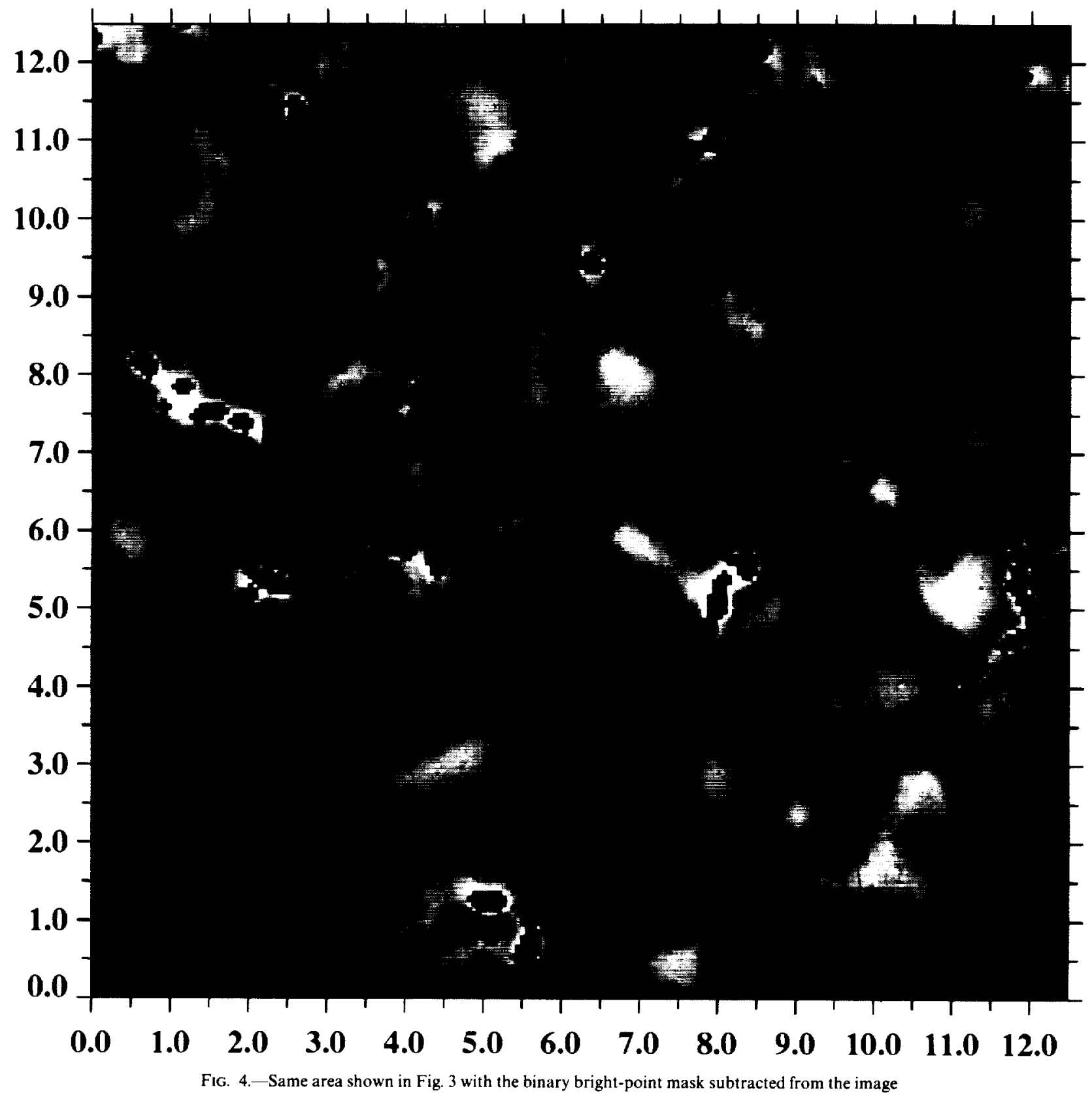

Berger et al. (see 454, 533) 